\title{
1 Evolution of two-component quorum sensing systems
}

\author{
2 Marina Giannakara, Vassiliki Lila Koumandou \\ 3 \\ 4 Genetics Laboratory, Department of Biotechnology, Agricultural University of Athens, \\ 5 Athens, Greece \\ 6 \\ 7 Corresponding author: \\ 8 Vassiliki Lila Koumandou \\ 9 lera Odos 75, Athens 11855, Greece \\ 10 Email address: koumandou@aua.gr \\ 11
}




\section{Abstract}

13 Quorum sensing (QS) is a cell-to-cell communication system that enables bacteria to

14 coordinate their gene expression depending on their population density, via the detection of small molecules called autoinducers. In this way bacteria can act collectively to initiate processes like bioluminescence, virulence and biofilm formation. Autoinducers are detected by receptors, some of which are part of Two Component Signal Transduction Systems (TCS), which comprise of a (usually membrane-bound) sensor histidine kinase (HK) and a cognate response regulator (RR). Different $Q S$ systems are used by different bacterial taxa, and their relative evolutionary relationships have not been extensively studied. To address this, we used the KEGG database to identify all the QS HKs and RRs that are part of TCS and examined their conservation across microbial taxa. We compared the combinations of the highly conserved domains in the different families of receptors and response regulators using the SMART and KEGG databases, and we also carried out phylogenetic analyses for each family, and all families together. The distribution of the different QS systems across taxa, indicates flexibility in HK/RR pairing, and highlights the need for further study of the most abundant systems. For both the QS receptors and the response regulators, our analysis indicates close evolutionary relationships between certain families, highlighting a common evolutionary history which can inform future applications, such as the design of novel inhibitors for pathogenic QS systems.

\section{Keywords}

33 Quorum sensing

34 Two Component Systems

35 Histidine Kinase

36 Response regulator

37 Conserved domains

38 Molecular Evolution

39 Phylogenetics 


\section{Introduction}

The mechanism and importance of quorum sensing

Quorum sensing is a mechanism responsible for regulating a variety of group behaviors and interactions in bacteria, including pathogenicity, biofilm formation, mobility, sporulation, conjugal plasmid transfer, bioluminescence, resistance to antibiotics, and antibiotics production (Bandara et al., 2012; Ng and Bassler, 2009). All quorum sensing systems, despite in their molecular mechanisms and regulatory components, regulate their target genes in a population-density dependent manner. An extracellular molecule (autoinducer, Al) is secreted by a group of bacteria and when the number of the bacteria rises, so does the number of these molecules produced in the surrounding area. When the density of the Als overcomes a specific threshold, they bind to a receptor protein, either on the membrane or in the cytoplasm of the cell. The detection of the Als activates a signal transduction cascade, altering the gene expression of the cells all over the population $(\mathrm{Ng}$ and Bassler, 2009).

The quorum sensing mechanism is different between Gram-positive and Gramnegative bacteria. Gram-positive bacteria use modified oligopeptides as Als (autoinducing peptides, AIPs), which bind to membrane-bound receptors - Histidine Kinases (HKs) - when they exceed a density threshold. This leads to the phosphorylation of the HK protein and its cognate response regulator (RR), which then regulates the target genes. The AIPs can also be transported into the cell and interact with cytoplasmic AIP receptors, which act as transcription regulators to regulate gene expression (Ng and Bassler, 2009; Rutherford and Bassler, 2012). Gram-negative bacteria use AHLs (acyl-homoserine-lactones) or SAMproducts (S-adenosylmethionine)(Papenfort and Bassler, 2016). A second group of Als are DPD (4,5-dihydroxy-2,3-pentanedione) derived molecules, collectively known as Al-2 (Bandara et al., 2012). Those small molecules can diffuse through the cell membrane and bind to LuxR- type cytoplasmic receptors, which function as transcription factors. However, in some Gram negative bacteria the Als are detected by Histidine Kinases and the process is similar to that in the Gram positive bacteria (Rutherford and Bassler, 2012). Other inducer molecules taking part in quorum sensing include protein molecules that are not described as AIPs, pheromones, sulfate and phosphate, fucose, oxygen, quinolones, $\gamma$-aminobutiric acid (https://www.genome.jp/kegg-bin/show_pathway?map02024) or even adrenaline and noradrenaline produced by the gut bacteria in humans, known as Al-3 (Reading and Sperandio, 2006).

Since quorum sensing affects many bacterial processes, it is an interesting subject of study which can have numerous applications, most important of which is the control of pathogenicity via QS inhibition, given the increasing spread of antibiotic-resistant bacteria. It has been suggested that targeting QS imposes less selective pressure, since it does not kill bacteria but only hinders the production of virulence factors (Whiteley et al., 2017). QS inhibition (Quorum Quenching, QQ) can be applied to fields such as hospital infections, phytopathogen control in agriculture and for producing new preservatives in the food 
industry (Sreenivasulu, 2015; Tiwari et al., 2016). The gene products involved in QS mechanisms are consequently possible targets in new antimicrobial strategies and the investigation of their evolutionary relationships can contribute to that direction (Lerat and Moran, 2004).

\section{Two-Component System types}

The Histidine Kinases that contribute to QS in both Gram positive and negative bacteria are part of the Two Component Signal Transduction (or Component) Systems (TCSs). TCS is the main mechanism in bacteria for responding to environmental stimuli and prevails across the entire bacterial kingdom (Capra and Laub, 2012). Although there is a wide variety in TCS mechanisms, they all fall into two main categories: The canonical and the multi-step TCS.

\section{Canonical TCS: His-Asp}

A typical TCS comprises two proteins: a transmembrane Histidine Kinase (HK) and a Response Regulator (RR). The HK is a dimeric protein which includes 3 domains: The Sensor, the HisKA (Histidine Kinase domain or Dimerization domain) and the HATPase (ATP kinase binding domain). The sensor domain binds to the Al and this interaction results in an ATPdependent autophosphorylation of the conserved histidine of HisKA. The phosphoryl group is then transferred to a protein called Response Regulator (RR). The RR contains the Response Regulator domain (or REC or Receiver domain) in the N-terminal region and the Effector domain in the C-terminus (Jacob et al., 2014; Kaserer and West, 2010). The first domain includes the conserved aspartate residue, which is phosphorylated (Kaserer and West, 2010). This leads to conformational changes in the structure of the RR (West and Stock, 2001), which is responsible for controlling quorum sensing-associated behaviors through the Effector domain either via protein-protein interactions or by binding to DNA for gene regulation (Mascher et al., 2006).

\section{Multi-step TCS: His-Asp-Asp}

A more complicated TCS mechanism usually includes a hybrid HK (HHK), a histidine phosphotransferase (HPt) and its cognate RR. The structure of the HHK is similar to HK but additionally includes a conserved Asp residue in the C-terminus, namely a REC domain. The binding of the signaling molecule induces the autophosorylation of the His of the HiskA domain and then the phosphoryl-group is transferred intramolecularly to the Asp residue of the HHK. Then the phosphotransfer continues to the HPt protein and from there to the RR (Capra and Laub, 2012; Kaserer and West, 2010).

\section{Categories of HKs and RRs}

The classification of the HKs and the RRs into groups varies and depends on the comparison criteria. In the KEGG database the HKs and their cognate RRs are divided into 
118 separate families (Supplementary Fig. S1); however, the criterion for this classification is not

119 obvious. In previous studies, the HKs and RRs were classified based on their conserved

120 domains. For example, the HKs were divided into 11 subfamilies, based on multiple

121 alignment of $348 \mathrm{HKs}$ and comparison of the "Homology boxes", i.e. groups of highly

122 conserved amino acids, which are assumed to be important in substrate binding, catalysis or

123 the structure of the protein: the $\mathrm{H}$ - and $\mathrm{X}$-boxes are parts of the HisKA domain, and the $\mathrm{N}$-, D-

124 F-and G-boxes are part of the HATPase_c domain; the D- box is also part of the REC domain

125 (Grebe and Stock, 1999). Alterntively, given the structure of the proteins and more

126 specifically their domain organization, the HKs can be divided into 4 groups: 3 groups with

127 proteins containing the intracellular domains PAS, HAMP or GAF, respectively, and a group

128 with none of these domains (Capra and Laub, 2012). In a more recent study, the proteins

129 were separated into 5 types (Types I-IV and the chemosensor HKs group, CheA) based on the

130 inferred phylogenetic tree using the whole genome of 22 bacterial species and 4 archaea.

131 More specifically, the criterion for their division was the $\mathrm{H}$-box (HisKA) and its secondary

132 structure, and the kinase domain (ATPase) (Forst and Kim, 2015).

133 Based on the DNA-binding domains (Effector domain) of RRs, they were classified into

134 three subfamilies: OmpR, Fix J and NtrC (Hakenbeck and Stock, 1996), or more recently into

1355 groups (Capra and Laub, 2012). By comparing the Receiver domains (REC), the RRs were

136 classified into 8 subfamilies $\left(R_{A}-R_{H}\right)$ (Grebe and Stock, 1999), however, it has been proposed

137 that the comparison of the RRs for their classification should be based only on the Effector

138 domain, due to the fact that the Receiver domains of different RRs are highly conserved and

139 therefore not very informative for classification (Galperin, 2006).

\section{Evolution of TCS}

141 It is suggested that the more variable the environment, the higher the number of the

142 TCS of the organisms living in it. The genes of the HKs and their cognate RRs are usually

143 found on the same operon. The various TCS pathways are the result of lateral gene transfer

144 and/or duplication. The duplication can happen to all of the genes of the operon or to one of

145 them; in the second case, the resulting TCS pathway consists of more than one HK and

146 responds to more than one extracellular signal, or it includes more than one RR and gives

147 multiple responses for a specific signal. Also, due to the modular nature of HKs, domain

148 shuffling can lead to new HKs and the fusion of the HK and RR genes of an operon can create

149 hybrid HKs. The function of TCS pathways relies on molecular recognition and consequently

150 the amino acids have extensively co-evolved as a means to prevent the disruption of

151 signaling by mutations. Co-evolution also prevents crosstalk with other pathways.

152 Concerning the origin of HKs, it has been suggested that they come from ATPases of the

153 GHKL superfamily: Hsp90, the mismatch repair protein MutL or type II topoisomerases

154 (Capra and Laub, 2012; Koretke et al., 2000). On the other hand, the origin of the RRs

155 remains unclear beyond a general structural similarity to P-loop NTPases (Koretke et al.,

156 2000). The evolution of RRs can be the result of changes in the DNA-binding or RNA-

157 polymerase interaction sites; it includes lateral gene transfer and gene duplication, while 
158 domain shuffling and rearrangement have also led to new forms of RRs (Capra and Laub, 159 2012).

160 Evolution of QS

161 Most of the phylogenetic studies of QS have mainly focused on the produced Als or on 162 specific pathways, mainly Luxl/LuxR and LuxS/LuxQ. A phylogenetic analysis for the proteins 163 Luxl, LuxR and LuxS, showed that these proteins are ancient in many bacterial species and 164 that they appeared very early in the evolutionary path of the bacteria. It was also found that 165 in most cases the gene pairs of the inducer and its cognate response regulator are located 166 next to each other on the chromosomes and they preserve their pairwise function, a sign of 167 common evolutionary history (Lerat and Moran, 2004). The existence of homologous 168 proteins in some genomes is representative of horizontal transfer and duplication of the 169 genes, both of which can alter the regulation of different gene targets (Lerat and Moran, 170 2004). In a study of the ComQXPA pathway, the phylogenetic tree of HK proteins in 60 171 firmicute genomes containing the comQXPA locus clustered all the ComP proteins from 172 various organisms together, instead of gathering them with HKs of the same organism.

173 Therefore, it concluded that ComP evolved from the other HKs before the appearance of the 174 modern bacterial species and thus the ComQXPA pathways have an ancient origin (Dogsa et 175 al., 2014).

176 In a study of the Vibrionaceae family, it was found that the various inducers (AHLS) do 177 not show any correlation with the species' geographical distribution. This result indicates 178 that AHLs had a worldwide distribution throughout their evolution, since there was no 179 identification of specific AHL to the environment of the organism which produces it 180 (Rasmussen et al., 2014). Another survey, focusing on thermophilic bacteria, showed that 181 they use Als-2 for QS communication. The phylogenetic tree comparison of LuxS and the 16S 182 rRNA of thermophiles and mesophiles show that LuxS (which produces the autoinducer) of 183 mesophilic bacteria may originate from thermophiles. Also, the LuxS proteins of 184 thermophilic bacteria within a phylum were evolutionarily closer compared to LuxS of 185 different phyla (Kaur et al., 2018).

186 In this study we examine the evolutionary relationships between different TCS QS 187 systems. To achieve this, we conducted phylogenetic and comparative analysis of the 188 components of these pathways. We used the KEGG database to identify all of the QS 189 proteins that are also part of the TCS, and we collected their amino acid sequences from 190 various bacterial species. The KEGG and SMART databases were used to compare the 191 combinations of highly conserved domains in HKs and RRs. Phylogenetic analysis was based 192 on Maximum Likelihood methods. 


\section{Methods}

195 Distribution of QS proteins

196 We used the KEGG Database (Kyoto Encyclopedia of Genes and Genomes,

197 https://www.kegg.jp/ or https://www.genome.jp/kegg/), to select the amino acid sequences

198 used in this study. First, the Two Component System protein types that are also part of the

199 Quorum Sensing system were found using the pathway maps of the KEGG PATHWAY

200 database (maps k002020 and ko02024 respectively). Each protein type is attributed to a

201 specific orthology group accession number. By entering each identifier in the KEGG

202 ORTHOLOGY database section, we were able to gather information about the amino acid

203 sequences of each protein type in different bacterial organisms ("Genes" section) and the

204 lineages in which each protein type is present ("Taxonomy"). We checked for the presence

205 of the protein subunits of different QS pathways in selected groups of bacteria, including

206 various Phyla, Classes and Genera, as shown in Supplementary Table S1.

\section{Conserved domains}

208 One amino acid sequence for each protein type was chosen randomly to identify the

209 conserved domains on it, by searching in the "motifs" section in the KEGG GENES database.

210 We also used SMART (Simple Modular Architecture Research Tool, http://smart.embl-

211 heidelberg.de/) to identify additional domains in our selected amino acid sequences not

212 shown in KEGG. Both SMART and KEGG also give the E-value of each conserved domain; the

213 domains that were finally taken into consideration for our research, were those with an E-

214 value of at least $10^{-5}$ which were also mentioned as "Confidently predicted domains" in

215 SMART. In general, most of the domains were the same in the two databases. Next, using

216 the Pfam database (https://pfam.xfam.org/), we found the groups (clans) in which these

217 domains are classified (Supplementary Table S2) as well as information about their function.

218 For further information we used the Conserved Domain Database (CDD) in NCBI

219 (https://www.ncbi.nlm.nih.gov/Structure/cdd/cdd.shtml).

220 Taxa and families representation

221 For the phylogenetic analysis, the number of the collected amino acid sequences for

222 each protein type was kept as low as possible so as to have a manageable amount of data

223 but at the same time include a representative portion of the different taxa and families

224 which include each protein type. As a result, the average number of the amino acid

225 sequences for each protein type was about 20. Supplementary Tables S3A and S3B show the

226 representation percentages of the taxa, families and species out of the total available ones

227 in the KEGG database. Accession numbers and abbreviations for all sequences used are in

228 the Supplementary dataset. 
Phylogenetic analysis

Multiple alignments of the amino acid sequences were created using the MAFFT (Multiple Alignment using Fast Fourier Transform, https://www.ebi.ac.uk/Tools/msa/mafft/) (Madeira et al., 2019) and MUSCLE (MUltiple Sequence Comparison by Log- Expectation, https://www.ebi.ac.uk/Tools/msa/muscle/) (Edgar, 2004), alignment programs, with default settings, for:

- each HK family

- all the HKs

- each RR family

- all the RRs

The substitution model for each multiple alignment was inferred via the ProtTest3.4 program (https://github.com/ddarriba/prottest3/releases) (Pearson, 2013). In order to compute the likelihood scores, the following settings were used:

- Substitution matrices to test: VT, Blosum62, JTT, Dayhoff, WAG

- Rate variation: I, G, I+G

- Amino acid frequencies: unchecked empirical

The results were evaluated by the Akaike information criterion (AIC). More specifically, the substitution model for the MUSCLE multiple alignments of the HKs and RRs was VT and for the MAFFT alignments was VT for HKs and WAG for RRs.

Phylogenetic reconstruction for each protein family was done with PhyML at http://www.phylogeny.fr/ (also available at https://ngphylogeny.fr/) (Dereeper et al., 2008). We used the "A la Carte" function, with the following settings: "remove gaps in alignment" (alignment curation), Maximum Likelihood method (PhyML), SH-like Approximate Likelihood-Ratio Test (aLRT) as the statistical test for branch support and 100 bootstraps. For each multiple alignment we chose the WAG substitution model as the best one according to the ProtTest results (WAG, JTT and Dayhoff were the given options in the PhyML settings).

The phylogenetic trees for all the protein families together ( 2 trees: HKs and RRs) were constructed in the CIPRES Science Gateway (https://www.phylo.org/portal2/login.action), using RAxML (Randomized Axelerated Maximum Likelihood): RAxML-HPC BlackBox (8.2.12) Phylogenetic tree inference using maximum likelihood/rapid bootstrapping on XSEDE (Stamatakis, 2014). Before entering the multiple alignments into the program, we removed any positions not conserved in $>50 \%$ of the sequences. For this purpose, we used Jalview (https://www.jalview.org/) to detect those positions and then the Mesquite program to do the trimming (http://www.mesquiteproject.org/). The proper substitution model was chosen for each multiple alignment and the bootstrap number was set to 100 .

The multiple alignment for all the HK proteins consisted of 347 sequences and had a total length of 2410 amino acids using MAFFT or 1919 using MUSCLE. In both alignments, there were several clusters of conserved residues mixed with gaps or non-conserved residues towards the N-terminal region of the alignment (41-867) (34-1073, 1295-1781) and near the C-terminal part (949-1411). The trimmed MAFFT alignment had 520 amino acids 
269 (Supplementary Fig. S2), while the trimmed MUSCLE alignment had 455 amino acids

270 (Supplementary Fig. S3). The multiple alignment for all the RR proteins consisted of 293

271 sequences and had a total length of 751 amino acids using MAFFT or 670 using MUSCLE.

272 Both the MUSCLE and MAFFT alignments contained conserved clusters alternating with gaps

273 or non-conserved residues (65-224, 287-528). Trimming resulted in an alignment of 229

274 amino acids using MAFFT (Supplementary Fig. S4), and 274 using MUSCLE (Supplementary

275 Fig. S5).

276

277

All the phylogenetic trees were visualized with the Figtree program (https://github.com/rambaut/figtree/releases). The results were saved as PNG images and were further edited with Microsoft Draw. Nodes were labelled as white, grey or black bullets, if their bootstrap value was 50\%-80\%, 80\%-95\% and 95\%-100\% respectively. The raw and masked alignments, as well as the phylogenetic trees in nexus format are available in the Supplementary dataset. The alignments for each family and all families together are available in the files "HK alignments" and "RR alignments". The alignments of all HKs and all RRs are presented as they were trimmed in Mesquite: in the added taxon, " $C$ " and "I" represent the removed and included positions. The trees in nexus format are available in the "HKs trees" and "RRs trees" files.

\section{Results}

Distributions of QS proteins

The distribution of each protein type across bacterial taxa is demonstrated in Fig. 1; bacterial groups are colored based on (Jun et al., 2010). All pathways are fully present in at least one bacterial group, but many groups of bacteria have some of the subunits of each pathway. All Lux pathways are fully present in $\gamma$-proteobacteria. The Lux system contains a pathway of HKs and an RR, and the cytoplasmic Lsr proteins; both pathways are activated by the Al produced by LuxS. Deinococci, actinobacteria, clostridia, bacilli, $\alpha$ - and $\gamma-$ proteobacteria, and Spirochaetes possess most or all proteins of the Lsr protein pathway. The UvrY pathways (BarA/UvrY and SdiA) are fully present in Y-proteobacteria. Like in the Lux pathway, there are two different types of receptors, an HK (BarA) and a cytoplasmic protein (SdiA). Several proteins of one or both of the pathways are also found in $\alpha-\beta$-and $\delta$ proteobacteria, chlorobi, bacilli, Nitrospira, Chrysiogenetes and Spirochaetes. Concerning the NarL pathway, $\gamma$-proteobacteria have all proteins of the pathway; $\alpha$ - and $\beta$ proteobacteria, chloroflexi, cyanobacteria, actinobacteria, clostridia and bacilli have one of the receptors. Only $\mathrm{Y}$-proteobacteria possess all proteins of the Rcs pathway; bacteroidetes and $\beta$-proteobacteria contain the HK and RR of the pathway. All three proteins of the Fus pathway are in $\gamma$-proteobacteria; however, the HK/RR pair is found also in $\alpha$ - and $\beta$ proteobacteria, bacteroidetes, deinococci, actinobacteria and Spirochaetes. All proteins of the Com(XQPASK) pathway are found in bacilli and most proteins are found in clostridia. 
308 all found in bacilli, whereas the HK/RR pair ComD/ComE is present in clostridia. The Agr

309

310

311

312

313

314

315

316

317

318

319

320

321

322

323

324

325

326

327

328

329

330

331

332

333

334

335

336

337

338

339

340

341

342

343

344

345

346

pathway (both AgrC/AgrA and SaeS/SaeR) is fully present in bacilli, while $\alpha$-proteobacteria and clostridia contain the Agr proteins only. All the proteins of the Nis pathway are present in both clostridia and bacilli, while bacteroidetes, chloroflexi and actinobacteria contain all but two of the proteins. Other bacterial groups contain parts of the pathway but not the full sensing system (they lack either one or both the HK and RR). Bacteroidetes, actinobacteria, clostridia and bacilli contain both the $\mathrm{CiaH}$ and CiaR proteins. Concerning the Qse pathway, $\gamma$-proteobacteria contain all of the proteins (QseC/QseB and QseE(GIrK)/QseF(GIrR)).

QseC/QseB are present in bacteroidetes, actinobacteria and aquificae.

QseE(GIrK)/QseF(GIrR) are found in $\beta$ - and $\delta$-proteobacteria and Nitrospira. No bacterial

group contains all the proteins of the Rpf pathway: Nitrospira, $\alpha$ - and $\beta$-proteobacteria contain the HK/RR pair RpfC/RpfG. All three proteins of the DesR pathway are present in $\alpha-$ ,$\beta$ - and $\psi$ - proteobacteria, actinobacteria and bacilli. For details of all other bacterial groups which contain only certain proteins of each pathway, please refer to Fig. 1.

Fig. 1 shows that the majority of the complete pathways are found in $\alpha-, \beta$ - and $\gamma^{-}$ proteobacteria, clostridia and bacilli. A significant number of proteins are also found in $\delta$ and $\varepsilon$-proteobacteria, bacteroidetes and actinobacteria. Also, based on the number of bacterial groups which contain proteins of each pathway, the most widespread proteins are those of the UvrY, DesR, Fus, Nis pathways and the Lsr proteins of the Lux pathway. A more imited distribution across taxa is seen for the proteins of the two Com pathways and the Rcs and Agr pathways. Looking at each protein separately the most widespread proteins are CsrA, QseB, DesA, FusR, malt and LuxS (Fig. 2). Interestingly, those belong to different pathways (CsrA: UvrY, FusR: Fus, QseB: Qse, DesA: DesR, malt and LuxS: Lux pathway). The Lux and UvrY pathways contain the most widespread as well as some of the most limited protein types (LuxQ, Qrr and CsrB). There is also a difference in the distribution of the HKs and their cognate RRs: In most cases, the RRs are found in more bacterial groups than their cognate HKs (NarL, ComA, NisR, FusR, QseB, GlrR, and RpfG).

Conserved domains

In the Pfam database, the domains are organized into larger groups called "clans". The NCBI database characterizes most domains as "superfamilies". Table 1 contains the clans and their domains found in the studied amino acid sequences and Supplementary Table S2 shows the accessions for each domain in PFAM and InterPro, as well as the entry of the superfamily (NCBI-CDD) and pfam clan they belong to. The following domains do not belong to any clan: MASE1, PilJ, Hpt, Sigma54_AID, Trans_reg_C

Conserved domains in HKs: The length of the HKs varies from 406 (AgrC) to 949 (RcsC) amino acids. The longest HKs are the hybrid ones (NarL family: BarA, RcsC, Lux family: LuxN, LuxQ and CqsS, "Other": RpfC) which also contain more conserved domains. Differences in the length of the sequences can also be found within the proteins of the same family. The HKs LuxQ and RcsC function as a complex with another protein (LuxP and RcsF, respectively). 
347 Most HKs contain 1-3 conserved domains and up to 6 (Fig. 3). Comparing the number of the

348 conserved domains, the members of the families OmprR, LytTR and NtrC (GIrK) have the

349 lowest number of conserved domains (1 or 2). In contrast, the HKs of the NarL, Lux and

350 "Other" (RfC) families have more than 2 conserved domains (Fig.3).

351

352

353

354

355

356

357

358

359

360

361

362

363

364

365

366

367

368

369

370

371

372

373

374

375

376

377

378

379

380

381

382

383

384

385

386
The common trait is the existence of either HATPase_c or HATPase_c_5, which are also the only conserved domains in the protein NisK and the proteins of the LytTR family. According to NCBI-CDD, HATPase domains (Histidine kinase-like ATPase domains), also referred to as GHKL ATPase domains, are found in ATP-binding proteins, including histidine kinases. HAMP (Histidine kinase, Adenylyl cyclase, Methyl-accepting protein, and Phosphatase domain) is the second most frequent domain, found in HKs of the NarL family ( NarX, NarQ, BarA). It relays various input signals but no common mechanism of HAMP has yet been established (NCBI-CDD). According to InterPro, one or more copies of the domain can be found in association with other domains, including HisKA. It has been suggested that its role is the regulation of the phosphorylation or methylation of homodimeric receptors by transmitting the conformational changes in periplasmic ligand-binding domains to cytoplasmic signalling kinase and methyl-acceptor domains. According to a study of Koretke et al., HAMP is the "linker region" and it is widely conserved in HKs as well as in chemoreceptors, bacterial nucleotidyl cyclases, and phosphatases. Its wide distribution is notable, as about a quarter of the HKs in the SMART database contain this domain and about half of all HAMP domains are found in HKs (Koretke et al., 2003). The Response_reg and Hpt domains are located in the C-terminal region of hybrid HKs, but only Hpt can alternatively be found as an independent protein (within the Rcs pathway). The Response_reg (Response regulator receiver domain or phosphoacceptor receiver-REC) receives the signal from the HK and $\mathrm{Hpt}$ (histidine-containing phosphotransfer domain), which mediates phosphotransfer reactions in Multi-step TCS signaling systems. Concerning the $\mathrm{N}$-terminal region, only few proteins contain conserved domains, most of which belong to the NarL family (QseC, NarX, NarQ, FusK). These are Cache-like sensory domains (2CSK_N, DUF2222 and LuxQ-periplasm), PilJ (Type IV pili methyl-accepting chemotaxis transducer), Peripla_BP_4 (Periplasmic_Binding domain) and MASE1 (PFAM: predicted integral membrane sensory domain in HKs, diguanylate cyclases and other bacterial signaling proteins).

Conserved domains in RRs: The average length of RRs is significantly shorter that the HKs. It varies from 207 to 455 amino acids. Most RRs contain 2 conserved domains except for LuxO, which has 3 domains (Fig. 3). The $\mathrm{N}$-terminal regions always contain the Response_reg domain, which is a common structure trait for all the RRs. The conserved domain on the Cterminal region is different in each RR family. The only exception is the protein GlrR ( $\mathrm{NtrC}$ family), which has the same conserved domain as LuxO (Lux family). An overview of the conserved domains of both HKs and RRs is present in Table 2. HATPase_c is the most common conserved domain, as it is found in 15 protein types. HisKA is the second most common, with 9 occurrences.

Concerning the variety of the Effector domains, the majority has a DNA-binding 
387

388

389

390

391

392

393

394

395

396

397

398

399

400

401

402

403

404

405

406

407

408

409

410

411

412

413

414

415

416

417

418

419

420

421

422

423

424

activity: HTH_8, Sigma70_r4, Sigma70_r4_2, GerE, and Sigma54_DBD (DNA-Binding Domain) are HTH (Helix-Turn-helix) domains. Although Sigma54_CBD (Core Binding Domain) is also considered as an HTH domain, it interacts directly with the core RNA polymerase, forming an enhancer-dependent holoenzyme. The centre of this domain is slightly similar to a HTH motif, which may represent a DNA-binding domain. Also, some domains are P-loop-NTPases: Sigma54_activat, Sigma54_activ_2 interact with the sigma-54 factor of RNA polymerase; AAA and AAA_5 (ATPases Associated to a variety of cellular Activities) are described as a dynein-related subfamily and although their role is yet unclear, the AAA+ superfamily to which AAA ATPases belong, is associated with chaperone-like functions. Sigma_54_AID (Sigma_54_Activator interacting domain) is not classified into any clan. It is necessary for the interaction of Sigma54 RNAP holoenzyme with the activator and it can also inhibit transcription initiation prior to interaction with the activator.

Phylogenetic trees by family

Given the families formed by the HKs and their cognate RRs, we constructed four phylogenetic trees for the HKs and three for the RRs. The HKs GlrK (NtrC family), RpfC ("Other"), their cognate RRs GlrR and RpfG, as well as LuxO are the only members of their family, hence no phylogenetic tree was inferred. Unless otherwise stated, only wellsupported clades, with $>90 \%$ bootstrap support, are discussed below. Phylogenetic reconstructions were done with PhyML using MAFFT alignments, but the major differences between the trees created from the MAFFT and MUSCLE alignments (Supplementary Fig. S6S9) are also mentioned, wherever they apply.

\section{The NarL family}

The HKs of the NarL family are organized in distinct clades in the PhyML tree (Fig. 4A). Each clade consists of amino acid sequences of the same protein type, supported by high bootstrap values. The same structure is observed in the phylogenetic tree of their cognate RRs (Fig. 4B); the clades of the RR types are also supported by good bootstrap values, but most of them are below $95 \%$. Although both trees have some amino acid sequences away from the clade of their protein type (e.g. marrComP, mdnBarA, paejNarQ and anxNarX), the phenomenon is more prevalent in the RRs. Notably, the structure of the phylogenetic tree shows three pairs of HK types (RcsC-BarA, ComP-FusK and NarQ-NarX), which group closer to each other than to the other protein types. This pattern is also seen for their cognate RRs.

The clade of the RcsC proteins is closer to BarA than the rest of the protein types. On the other hand, their cognate RRs (RcsB and UvrY) are apart in the PhyML tree: although the UvrY proteins do not create a distinct pair with any other RR type, their clade is closer to FusR and the common clade of NarL and NarP. The RcsB RRs group together, except for the sequence abamRcsB. The UvrY proteins also form a clade ( $86 \%$ bootstrap support, excluding the first node of maesUvrY, nppNarL and ssilNarP sequences); outside of it sbxUvrY, dunUvrY and cbaeUvrY fall in the RcsB clade, mmarUvrY falls in the NarL clade, and tjeUvrY in the 
425 NarP clade. A comparison with the same PhyML tree from the MUSCLE alignment

426 (Supplementary Fig. S6B) shows that although the distribution of the NarL and NarP RRs is in

427 multiple dispersed clades in the tree, the UvrY and RcsB proteins clearly form a common

428 clade (87\% bootstrap value). Also, the ComA clade is closer to FusR than to RcsB.

429 The ComP clade has a $50 \%$ bootstrap value, excluding kbsComP and marrComP, and it 430 groups with the FusK clade (72\% bootstrap value). In contrast, the clades of their cognate 431 RRs, ComA and FusR, do not group together. The ComA sequences are not directly related to 432 any other clade but they are close to $R \operatorname{csB}$ in the tree.

The NarX proteins are separated in two main clades, both of which share a common node with the NarQ clade; spswNarX branches out earlier. However, the distribution of their cognate RRs NarL and NarP is more complicated. They are divided into several clades (sometimes including both protein types), yet most of these clades are placed close to each other and their common node has a 79\% bootstrap value. In detail, the NarP sequences are grouped into two distant main clades; a larger one, neighboring with the NarLs and a smaller distant one. There are more sequences outside of these clades than is seen for their cognate HK sequences, and from different species than in the HKs tree. The NarLs are gathered in several small clades close to each other, most of which demonstrate bootstrap values of at least $80 \%$, but many NarL sequences are dispersed throughout the phylogenetic tree.

The OmpR family

All different HK types of the OmpR family are grouped into separate clades. None of the HK sequences is misplaced in a clade of a different protein type, except spyaNisK, which falls in the CiaH clade. Their cognate RRs follow a similar pattern, except for sequences phmQseB and aprsQseB, which lie outside the QseB clade. There are two related groups in both phylogenetic trees: $\mathrm{CiaH}$ - QseC and NisK - SaeS and their cognate RRs, CiaR - QseB and NisR - SaeR (Fig. 5A and 5B). The majority of the CiaH sequences stem from a common node, with soxCiaH and cooCiaH branching out earlier. The QseC sequences are distinctly separated from the rest of the RR types and they share a common node with the CiaH clade. The cognate RRs follow the same pattern: The clade of the QseB sequences (85\% bootstrap value) forms a common clade with the CiaR sequences. The NisK sequences are organized into one main clade and they have a common node with all the SaeS proteins. The NisR proteins also group together with the clade of the SaeR sequences.

\section{The Lux family HKs}

The sequences of each HK type of the Lux family (LuxN, LuxQ and CqSS) are grouped into distinct clades (Fig. 6). The LuxN sequences have one common node. On the other side of the tree, LuxQ create a common clade, although dhyLuxQ, dsaLuxQ, oceLuxQ are more 
462 (Supplementary Fig. S8) appear near the core of the tree; it seems that the clades CqsS and

463 LuxQ switch positions in the MAFFT and MUSCLE versions of the tree. As there is only one RR

464 type for all the Lux family HKs (LuxO), there is no phylogenetic tree for the RRs of the Lux

465 family.

466 The LytTR family

467 The different types of HKs of the LytTR family, as well as their cognate RRs, are clearly 468 separated from each other and both phylogenetic trees have a similar overall appearance 469 (Fig. 7A and 7B). The AgrC sequences form several clades, all of which stem from a common 470 node on the phylogenetic tree. The two AgrC2 sequences are found in a distinct clade within 471 the AgrC clade, closest to rimAgrC. Similar to AgrC HKs, the AgrA sequences do not form one 472 distinct clade, but smaller ones, gathered on one side of the phylogenetic tree. The AgrA2 473 sequences are found in a distinct clade near the AgrA clades closest to cdfAgrA ( $77 \%$ 474 bootstrap value). Most of the ComD sequences are gathered in one clade, apart from the 475 IhiComD, IsjComD and laliComD sequences, which are placed among AgrC sequences. The 476 ComE RRs create a main common clade, with the exception of an earlier smaller clade: 477 sequences std2ComE and smb1ComE branch early ( $58 \%$ bootstrap support) as do their 478 cognate HKs.

479

Phylogenetic trees of all the families together

\section{Phylogenetic analysis of all the HK families}

482

The two phylogenetic trees of all the HKs and RRs families together are discussed below, based on the MAFFT alignment. Major differences with the phylogenetic trees based on the MUSCLE alignments are also mentioned. In general, the members of each HK family tend to form one main clade, although the bootstrap values are not always significant. The clades of each HK protein type are also mostly monophyletic (Fig. 8). The sequences of the LytTR family, as well as the RpfC ("Other") and GIrK HK types (NtrC family) are each gathered in one clade, whereas the protein sequences of the Lux as well as the NarL family are found in more than one clades. The following 3 groups of families are discernible: 1) LytTR and

491 NarL (NarQ, NarX, FusK and ComP), 2) OmpR and NtrC, 3) Lux, NarL (BarA, RcsC) and "Other" (RpfC).

492 NarL family: The sequences of this family form three main clades: the RcsC clade, the BarA 493 clade (97\% bootstrap value excluding the early branches) and a third -and larger- one which includes the NarQ, NarX, FusK and ComP sequences (Fig. 8). Within this larger group, the 495 FusK sequences cluster together (56\%) and create a common clade with the NarQ and NarP clades. All the ComP sequences also derive from a common node, but with very low

497 bootstrap value. NarQ sequences form a well-supported main clade, while NarX forms two 498 main clades with $87 \%$ and $97 \%$ bootstrap support, respectively. Only one NarQ and three 
499 NarX sequences fall outside their protein type clades. In general, the grouping of the clades

500 of the NarL family is very similar to the tree for the HKs of the NarL family alone (Fig. 4): the

501 BarA-RcsC, and NarX-NarQ pairs are recapitulated, and the only difference is the positions of

502 FusK, which is closer to ComP in Fig. 4, while it shares a common node with the NarQ/NarX

503 clade in Fig. 8.

504 OmpR family: The HK types of this family are separated into three main clades: one for QseC 505 and $\mathrm{CiaH}$ one clade for the SaeS and one for the NisK sequences (Fig. 8). The tree from the

506 MUSCLE alignment (Supplementary Fig. S10) recapitulates the exact structure of the

507 phylogenetic tree of the OmpR family alone (Fig. 5), as SaeS and NisK sequences create a

508 common clade. The clades of each protein type of the OmpR family are supported by high

509 bootstrap values, although their common node with the rest of the phylogenetic tree has

510 low bootstrap support.

511 Lux family: Two distinct clades and a smaller mixed one are formed by the HKs of the Lux

512 family: one clade consists of CqsS and LuxN sequences (with dhyLuxQ and dsaLuxQ at the

513 root of the clade) and neighbors with the OmpR, LytR and NarL clades and the other is made

514 of only LuxQ sequences near the BarA clade of the NarL family (Fig. 8). This pattern is also

515 seen in the tree based on the MUSCLE (Supplementary Fig. S10), and follows the groupings

516 of the phylogenetic tree of the Lux family alone (Fig. 6), as CqsS and LuxN are grouped

517 together, while the LuxQ sequences are placed away from the rest of the members of the

518 Lux family.

519 LytTR family: All the sequences of this family derive from a common node (56\% bootstrap

520 support) (Fig. 8). The ComD sequences form one clade (88\%) within the AgrC clade. The AgrC

521 sequences form many small clades, including the two AgrC2 sequences. The closest clade to

522 the LytTR family is the overall clade of the ComP-NarX-NarQ-FusK sequences of the NarL

523 family (62\% bootstrap support).

524 NtrC family (GIrK proteins-QseE): All the sequences of the GlrK (QseE) HK are in one clade,

525 with 73\% bootstrap support (Fig. 8). The closest clade to them is that of SaeS (of the OmpR

526 family), but with insignificant bootstrap support; in the tree based on the MUSCLE

527 alignment, the NtrC family groups more clearly with the OmpR family, and in particular the

528 common clade of QseC and $\mathrm{CiaH}$, although again with very low boostrap support.

529 "Other" (RpfC sequence): All RpfC sequences are placed in one clade, with 74\% bootstrap

530 value. The neighboring clade consists of RcsC sequences of the NarL family (Fig. 8), but the

531 bootstrap value for their common clade is very low.

532 
534 The RR families are distinctly separated from each other in the phylogenetic tree. Also, it is

535 clear that the sequences of each RR type within each family remain together, like in the

536 phylogenetic tree of each family. Very few sequences fall outside of the clade of their family

537 or RR type (Fig. 9). An overview of the tree reveals a closer relationship between the

538 following family groups: 1) the RpfG and NarL families, 2) the OmpR, NtrC and Lux families

539 and 3) the LytTR clade, which is closer to group 1). In the tree based on the MUSCLE

540 alignment (Supplementary Fig. S11), the position of the RpfG (Other) group is closer to NtrC

541 and Lux families; however, the position of this group relative to other families is not

542 supported by high bootstraps in either of the trees.

543 NarL family: The NarL family members create a sum of clades, each consisting mostly of

544 sequences of one particular NarL protein type. The positions of all the family members are

545 very close to each other, to the extent that there are no defined pairs of protein types. The

546 RcsB and ComA clades branch later in the NarL family. All RcsB and ComA RRs are grouped in

547 their own RR type clade, with the exception of abamRcsB. The clade of the UvrY sequences

548 has, in general, nodes of low bootstrap value and five sequences are placed outside of it:

549 tjeUvrY, dunUvrY and mmarUvrY. All the FusR RRs form one clade, except spirFusR and

550 sphnFusR, which are right before the clade of the RcsB proteins. Most NarPs are divided into

551 two main clades, one with NarL sequences and a second one near the ComA sequences. The

552 NarL sequences are widely distributed in the clades of the NarL family, forming several small

553 clades. One group of these clades is close to the NarP sequences and another group is placed

554 among the ComA and FusR clades.

555 OmpR family: The members of the OmpR family are grouped together ( $86 \%$ bootstrap value)

556 and there is one individual clade for each protein type, supported by significant bootstrap

557 values (except NisR: 63\%). SaeR and NisR group together with $72 \%$ bootstrap value and CiaR

558 with QseB (58\% bootstrap). This recapitulates the pattern seen in Fig 5B. Only phmQseB is

559 distant in the tree, near th RpfG clade (but with inisgnficant bootstrap support).

560 Lux family: The Lux family (LuxO) (78\%) is close to NtrC, forming a common clade (52\%). This 561 clade shares a common node with the OmpR family (69\%).

562 LytTR family: The clade for all the RRs of the LytTR family is closer to the NarL and RpfG

563 family members and it consists of many smaller clades or individual branches. The different

564 RR types belong to separate clades where the AgrA2 sequences branch out earlier of all and

565 they have a common node with the AgrA clade (100\%), wheras the ComE is the last clade.

$566 \quad$ NtrC family (GlrR protein): The GlrR sequences group together; however, the early nodes

567 have a very low bootstrap value. The obgGlrR sequence branches out early with a very low 
568 bootstrap value (32\%), while dyeGIrR lies outside the $\mathrm{NtrC}$ clade, on the LuxO clade, with

$56978 \%$ bootstrap value.

570 "Other" (RpfG protein): The RpfG RRs form one clade with 96\% bootstrap value, which

571 includes no sequences of other RR type. The closest RR family is NarL, complying with the

572 structure of the tree of all the HKs (Fig. 8).

\section{Discussion}

575 The QS mechanism includes a multitude of signal molecules and pathways, which aim

576 to coordinate the activities of a bacterial population by changing gene expression according

577 to the population density. The fact that this mechanism affects various processes makes this

578 mechanism a potential target in antibiotics research. Few phylogenetic analyses on the

579 bacterial QS exist, and given the multiple pathways contributing to QS, studies have so far

580 focused only on the signaling molecules, or on specific pathways. Among them, the Luxl/R

581 pathway (Gram positive) has been mainly studied. Based on the lack of a wider phylogenetic

582 study of QS proteins, we chose to analyze proteins of the QS pathways which belong to a

583 widespread signal transduction system, the Two Component System (TCS). This mechanism

584 includes pairs of a Histidine Kinase (HK) and its cognate Response Regulator (RR). The

585 studied QS pathways are found in a wide variety of both Gram-positive and Gram-negative

586 bacteria.

QS protein conservation

588 Looking at the distribution of the different QS pathways across bacterial taxa, the 589 overall conclusion is that $\alpha-, \beta-, \gamma$-proteobacteria, clostridia and bacilli possess the highest 590 number of the QS proteins (Fig. 1). The most widespread proteins are CsrA, FusR, QseB, 591 DesA, malt and LuxS. These belong to various pathways and families and they have different 592 roles, indicating no clear relationship between these characteristics and the number of the 593 bacterial groups containing them. The least widespread proteins are found in the two Com 594 pathways, the Rcs and Agr pathways, as well as the Lux pathway. These results suggest that 595 the wide presence of one protein doesn't mean a similar distribution for the rest of the 596 proteins of the same pathway. This contradiction is pointed out by the fact that the Lux and 597 UvrY pathways contain both the most widespread and some of the most limited protein 598 types (LuxQ, Qrr and CsrB). Also, there is a difference even in the distribution of the HK/RR 599 pairs, i.e. between the HK and its cognate RR. This distribution may simply be the result of 600 inadequacies in genome annotation, or it may be an indication of flexibility in the system, 601 meaning that HKs can function with other than their "cognate" RRs, in essence leading to 602 mixed pathways. Although TCS pathways demonstrate a high specificity overall, such "cross603 talk" between different TCS pathways is possible (Agrawal et al., 2016). In the case of 604 "orphan" HKs or RRs with no cognate protein (Freedman et al., 2019; Wang et al., 2009), an 
605 interaction between an orphan protein with a non-cognate one can also occur (Wang et al.,

606 2009). Also, given the QS map of KEGG (Fig. 3), QseC can interact with GlrR (QseF), which is

607 not only a non-cognate RR, but it also belongs to a different family (NtrC); interestingly, QseC

608 and GIrK both bind to Adrenaline and Noradrenaline. All of the interactions depicted in the

609 QS maps of KEGG (maps ko02020 and ko02024) involve proteins of the OmpR family. QseB

610 inhibits the cognate pair of Fusk/FusR of the NarL family, CiaR inhibits ComC, ComD and

611 ComE proteins of the LytTR family and SaeS is activated by the agr QS signal.

612 Examining each protein family of our study separately, it seems that the most

613 widespread HK/RR pairs of QS are those of the NarL, OmpR and NtrC families, whereas the

614 Lux and LytTR are the least common, although the Lux pathway is the most well-known and

615 well-studied QS pathway.

616

617 Conserved Domains

618 The HK-RR pairs belong to 6 families, as defined by KEGG: OmpR, LytTR, NarL, Lux, NtrC 619 and "Other". In both the OmpR and the LytTR family, the different protein types consist of a 620 small number of common conserved domains. On the other hand, HKs of the NarL and Lux 621 families show common domains and a number of variable domains; notably, the NarL and

622 Lux families also include hybrid HKs. The RRs of all families contain common domains (all of 623 them have the Helix-Turn-Helix motif).

624 The Lux family consists exclusively of Hybrid HKs, and all 3 of them activate a common

625

626 phosphotransfer protein and RR. The NtrC contains only the GIrK HK, which has the same

627 conserved domains with the HKs of the OmpR family, a characteristic which is also evident in

628 their close relationship on the phylogenetic tree. However, its cognate RR (GIrK) has various

629 common domains with the RR of the Lux family. This is also evident in the phylogenetic trees. Therefore, we conclude that similarities can be found not only among the protein types within a family, but also among different family members, which indicate a possible common evolutionary history.

There is partial agreement of our results with previous sudies on the classification of HKs and RRs. The differences are mostly due to differences in the selection of proteins

634 included in each study, and due to differences in classification based on domain structure 635 alone versus phylogenetic analysis. For example, looking at the classification of the HKs in 636 the Grebe and Stock study, ComP, NarQ and NarX were classified in group HPK7, CiaH in 637 HPK1a, NisK in HPK3c and BarA, RcsC, RpfC in HPK1b (Grebe and Stock, 1999). However, only 638 some of the HKs mentioned here were also included in that study, and this way of 639 classification differs from the KEGG database and our phylogenetic trees results, as $\mathrm{CiaH}$ and 640 NisK (OmpR family members) belong to separate groups and RpfC (hybrid HK) is placed with 641 the hybrid HKs of the NarL family (although hybrid HKs were placed in other HPK groups as 642 well). 
643

644

645

646

647

648

649

650

651

652

653

654

655

656

657

658

659

660

661

662

663

664

665

666

667

668

669

670

671

672

673

674

675

676

677

678

679

680

In the study of Forst and Kim, BarA and RcsC were characterized as Type IB and NarX and NarQ as Type III HKs (Forst and Kim, 2015). However, no members of other families included in our study were present in that analysis, so we cannot conclude if the comparison criteria used by Forst and Kim led to a different classification or if their classification is more detailed and separated the members of one family into multiple groups. Capra and Laub classified the HKs into 4 groups depending on the presence of PAS, HAMP and GAF domains or the absence of these domains (Capra and Laub, 2012). According to the authors, most HKs have at least one domain between the transmembrane part of the protein and the Histidine phosphotransfer domain, with PAS, HAMP and GAF being "by far the most common" ones. However, our studied proteins had no PAS and GAF domains, with the exception of RcsC, in which the PAS domain had a very high E-value in KEGG and SMART and thus it didn't meet our criterion to be included in the conserved domains of our study. Nevertheless, all three HKs with a HAMP domain (NarX, NarQ, BarA) were grouped to the same family (NarL), in agreement with the Capra and laub study. What contradicts their classification is the fact that the NarL family contains both HKs with HAMP and others without a HAMP domain, which are supposed to belong to different groups according to Capra and Laub. Similarly, the effector domains for the classification of the RRs were also different in the research of Capra and Laub, as no GGDEF and Methyltransfer domains were present in the proteins of our study. However, the LuxO and GIrR proteins possessing the AAA_5 domain, were grouped close to each other in our phylogenetic trees, complying with the classification criterion of Capra and Laub.

Concerning the classification of the RRs according to Hakenbeck and Stock (Hakenbeck and Stock, 1996) it is the same as the data of KEGG and our study. CiaR and NisR were classified to the OmpR family and ComA, NarP, NarL and RcsB were classified to the FixJ family. The results of Grebe and Stock are similar(Grebe and Stock, 1999): CiaR and NisR were classified to the $R_{A 1}$ group and ComA and RcsB to $R_{E}$, but NarP and NarL were classified to a different group $\left(R_{C 1}\right)$. In this paper, AgrA and ComE are also mentioned and grouped to $R_{D}$, both of which belong to the LytTR family in our study.

\section{Phylogenetic trees}

In all the inferred phylogenetic trees of each family, the amino acid sequences of both HKs and RRs are grouped in separate distinct clades according to their protein type and in many cases, with high bootstrap value. The same happens in the phylogenetic trees of all families together, with distinct clades for sequences of different family and protein types. Also, the relation among protein types (either HKs or RRs) is generally the same in the tree of each family and of all families together. In most cases, the relations among the HKs are also reflected by the relations of their cognate RRs, indicating a common evolutionary history, as has already been suggested by former studies on other proteins of the TCS (Koretke et al., 2000). 
681

682

683

684

685

686

687

688

689

690

691

692

693

694

695

696

697

698

699

700

701

702

703

704

705

706

707

708

709

710

711

712

713

714

715

716

717

718

719

720

The members of the NarL family form pairs of HKs that are closely related, NarX-NarQ, BarA-RcsC and ComP-FusK in both the tree of the NarL family alone and the tree of all the HK families together. In both trees the BarA-RcsC group is more distant. The tree for all the families together placed all RR types of the NarL family very close to each other: NarL and NarP clades are clustered together and UvrY sequences are placed close to RcsB, being in agreement with the trees of their cognate HKs. However, in the RR tree of the NarL family alone, only NarL and NarP demonstrate a similar grouping to the HKs from which they are activated: ComA is closer to RcsB (instead of FusR), and UvrY is closer to the clade of NarL/NarP (instead of RcsB).

The different protein types of the OmpR family demonstrate the same relation in all the phylogenetic trees (of the OmpR family alone and the tree with all the HK families together). There are two pairs of HKs: CiaH-QseC and SaeS-NisK. Their cognate RRs follow the same relation in all the RRs phylogenetic trees: the SaeR clade is closer to NisR and the CiaR clade is closer to QseB. Regarding the Lux HKs, both the tree of the Lux family and the tree for all the HKs show a similarity between the CqsS and LuxN. The latter also places the LuxQ sequences in the clade of other families (BarA, RpfC and $R \operatorname{cs} C$ sequences). Their common RR ( $L u x O$ ) is in the same clade with GIrR in the tree of all RRs. The protein members of the LytTR family have separate clades for the AgrC-ComD and ArgA-ComE sequences in all trees. The AgrC2 and AgrA2 sequences belong to the AgrC and AgrA clade, which is confirmed by all the inferred phylogenetic trees. The position of the RpfC/RpfG and GIrK/GIrR sequences (NtrC family) are slighlty different for the HKs and their RRs. The RpfC sequences are closer to RcsC, whereas RpfG is closer to NarL and NarP. GlrK is closer to the OmpR family but GIrR is in the same clade with LuxO.

A comparison of the phylogenetic trees of all HKs and all RRs suggests two clusters of the QS protein families: 1) OmpR-NtrC-Lux,and 2) NarL and LytTR, as well as clusters of protein types per family as outlined above. These evolutionary relationships highlight a common evolutionary history, and can inform future applications, such as the design of novel inhibitors for pathogenic QS systems. In fact, there have already been numerous scientific papers about Quorum Quenching, including for some of the proteins of our study. Diol-containing compounds, boronic acids and sulfones have been suggested as antagonists of the signaling molecules which bind with LuxP and as potential solutions to biofilm formation (Brackman and Coenye, 2014). Other molecules inhibiting the QS pathways of Gram-negative pathogens have been reported, including for the HKs and RRs of the Lux family and $\mathrm{RpfC} / \mathrm{RpfG}$, by blocking the receptors, inhibiting the production of the signaling molecules, or by degrading them (Defoirdt, 2018). The inhibition of the Agr pathway of Staphylococcus aureus has also been studied, with natural and synthetic substances targeting either AgrC or AgrA: the cyclodepsipeptide Solonamide B of Photobacterium halotolerans interferes with the binding of AIPs to AgrC, whereas $\omega$-hydroxyanodin from Penicillium restrictum binds to AgrA and therefore inhibits the expression of the regulated genes (Scoffone et al., 2019). Savirin (S. aureus virulence inhibitor) is another small molecule 
721 that selectively targets AgrA (Sully et al., 2014). The synthetic small molecule inhibitor

722 LED209 is selective to QseC (Curtis et al., 2014). The genes ComD (HK) and ComE (RR) was

723 also found to be downregulated by a small molecule containing a 2-aminoimidazole subunit

724 (2A4) along with five other biofilm-related genes of S. mutans (Liu et al., 2011). The

725 antiobiotic mupirocin when used in sub-inhibitory concentrations on high-level mupirocin-

726 resistant MRSA strains (Methicillin-resistant Staphylococcus) interferes with the agrA and

727 saeRS genes (Jin et al., 2018). Our prediction is that such inhibitors are more likely to also

728 interfere with protein types similar to the original target protein, as highlighted by the

729 phylogeneti analysis, than with more distant ones, e.g. an inhibitor for a Lux family HK may

730 also affect NtrC family HKs. On the other hand, pathway cross-talk in species with multiple

731 QS systems may hinder these inhibitory effects. Broader inhibition approaches have also

732 been suggested, with the ATP-binding domain of HKs (HATPase domain) being the main

733 target for TCS pathways inhibition (Wilke et al., 2015; Worthington et al., 2013).

734 Two-component signal transduction arose early in bacterial evolution after their

735

736

737

738

739

740

741

742

743

744

745

746

747

748

749

750

751

752

753

754

755

756

757

758 separation from the last common ancestor with significant diversification throughout the subsequent bacterial speciation; HKs arose from ATPases of the GHKL superfamily and pyruvate dehydrogenase kinases (PDKs), while the origin of the RRs remains unclear (Capra and Laub, 2012; Koretke et al., 2000). Regarding the origin of two-component quorum sensing systems, a phylogenetic analysis for the proteins Luxl, LuxR and LuxS in firmicutes, proteobacteria and actinobacteria, indicated that these proteins appeared very early in the evolutionary path of the bacteria, but with multiple occassions of horizontal gene transfer resulting in their present-day distribution (Lerat and Moran, 2004). In a study of the ComQXPA pathway in firmicutes, the phylogenetic tree of HK proteins clustered all the ComP proteins from various organisms together, instead of gathering them with other HKs of the same organism, indicating an early origin of ComP (Dogsa et al., 2014). The phylogenetic analyses presented here are unrooted and assume an early origin of these pathways in bacterial evolution. We present the distribution and evolutionary relationships of different HK and RR protein types and families, but a larger-scale analysis of all available sequences for two-component QS systems and other HK/RR pairs would be needed to confidently address the issue of the root of quorum sensing.

\section{References}

Agrawal, R., Sahoo, B.K., Saini, D.K., 2016. Cross-talk and specificity in two-component signal transduction pathways. Future Microbiol. 11, 685-697. https://doi.org/10.2217/fmb2016-0001

Bandara, H.M.H.N., Lam, O.L.T., Jin, L.J., Samaranayake, L., 2012. Microbial chemical signaling: A current perspective. Crit. Rev. Microbiol. 38, 217-249. https://doi.org/10.3109/1040841X.2011.652065 
759 Brackman, G., Coenye, T., 2014. Quorum Sensing Inhibitors as Anti-Biofilm Agents. Curr.

760

761

762

763

764

765

766

767

768

769

770

771

772

773

774

775

776

777

778

779

780

781

782

783

784

785

786

787

788

789

790

791

792

793

794

795 Pharm. Des. 21, 5-11. https://doi.org/10.2174/1381612820666140905114627

Capra, E.J., Laub, M.T., 2012. Evolution of Two-Component Signal Transduction Systems. Annu. Rev. Microbiol. 66, 325-347. https://doi.org/10.1146/annurev-micro-092611150039

Curtis, M.M., Russell, R., Moreira, C.G., Adebesin, A.M., Wang, C., Williams, N.S., Taussig, R., Stewart, D., Zimmern, P., Lu, B., Prasad, R.N., Zhu, C., Rasko, D.A., Huntley, J.F., Falck, J.R., Sperandio, V., 2014. QseC inhibitors as an antivirulence approach for gramnegative pathogens. MBio 5. https://doi.org/10.1128/mBio.02165-14

Defoirdt, T., 2018. Quorum-Sensing Systems as Targets for Antivirulence Therapy. Trends Microbiol. 26, 313-328. https://doi.org/10.1016/j.tim.2017.10.005

Dereeper, A., Guignon, V., Blanc, G., Audic, S., Buffet, S., Chevenet, F., Dufayard, J.F., Guindon, S., Lefort, V., Lescot, M., Claverie, J.M., Gascuel, O., 2008. Phylogeny.fr: robust phylogenetic analysis for the non-specialist. Nucleic Acids Res. 36, W465-W469. https://doi.org/10.1093/nar/gkn180

Dogsa, I., Choudhary, K.S., Marsetic, Z., Hudaiberdiev, S., Vera, R., Pongor, S., Mandic-Mulec, I., 2014. ComQXPA quorum sensing systems may not be unique to Bacillus subtilis: A census in prokaryotic genomes. PLoS One 9. https://doi.org/10.1371/journal.pone.0096122

Edgar, R.C., 2004. MUSCLE: Multiple sequence alignment with high accuracy and high throughput. Nucleic Acids Res. 32, 1792-1797. https://doi.org/10.1093/nar/gkh340

Forst, S., Kim, D., 2015. Genomic analysis of the histidine kinase family in bacteria and archaea. Microbiology 147, 1197-1212. https://doi.org/10.1099/00221287-147-5-1197

Freedman, J.C., Li, J., Mi, E., McClane, B.A., 2019. Identification of an important orphan histidine kinase for the initiation of sporulation and enterotoxin production by Clostridium perfringens type $F$ strain SM101. MBio 10, 1-17. https://doi.org/10.1128/mBio.02674-18

Galperin, M.Y., 2006. Structural classification of bacterial response regulators: Diversity of output domains and domain combinations. J. Bacteriol. 188, 4169-4182. https://doi.org/10.1128/JB.01887-05

Grebe, T.W., Stock, J.B., 1999. The histidine protein kinase superfamily. Adv. Microb. Physiol. 41, 139-227. https://doi.org/10.1016/s0065-2911(08)60167-8

Hakenbeck, R., Stock, J.B., 1996. Analysis of two-component signal transduction systems involved in transcriptional regulation. Methods Enzymol. 273, 281-300. https://doi.org/10.1016/s0076-6879(96)73026-4

Jacob, S., Foster, A.J., Yemelin, A., Thines, E., 2014. Histidine kinases mediate differentiation, stress response, and pathogenicity in Magnaporthe oryzae. Microbiol. Open 3, 668- 
796

797

798

799

800

801

802

803

804

805

806

807

808

809

810

811

812

813

814

815

816

817

818

819

820

821

822

823

824

825

826

827

828

829

830

831

832

687. https://doi.org/10.1002/mbo3.197

Jin, Y., Li, M., Shang, Y., Liu, L., Shen, X., Lv, Z., Hao, Z., Duan, J., Wu, Y., Chen, C., Pan, J., Yu, F., 2018. Sub-inhibitory concentrations of mupirocin strongly inhibit alpha-toxin production in high-level mupirocin-resistant MRSA by down-regulating agr, saeRS, and sarA. Front. Microbiol. 9, 1-8. https://doi.org/10.3389/fmicb.2018.00993

Jun, S.R., Sims, G.E., Wu, G.A., Kim, S.H., 2010. Whole-proteome phylogeny of prokaryotes by feature frequency profiles: An alignment-free method with optimal feature resolution. Proc. Natl. Acad. Sci. U. S. A. 107, 133-138. https://doi.org/10.1073/pnas.0913033107

Kaserer, A.O., West, A.H., 2010. Histidine kinases in two-component signaling pathways, in: Handbook of Cell Signaling, 2/E. Elsevier Inc., pp. 581-586. https://doi.org/10.1016/B978-0-12-374145-5.00078-4

Kaur, A., Capalash, N., Sharma, P., 2018. Quorum sensing in thermophiles $\square$ : prevalence of autoinducer-2 system. BMC Microbiol. 18, 62.

Koretke, K.K., Lupas, A.N., Warren, P. V, Rosenberg, M., Brown, J.R., 2000. Evolution of TwoComponent Signal Transduction. Mol Biol Evol. 17, 1956-1970.

Koretke, K.K., Volker, C., Bower, M.L., Lupas, A.N., 2003. Molecular Evolution of Histidine Kinases. Histidine Kinases Signal Transduct. 483-506. https://doi.org/10.1016/B978012372484-7/50024-2

Lerat, E., Moran, N.A., 2004. The Evolutionary History of Quorum-Sensing Systems in Bacteria. Mol. Biol. Evol. 21, 903-913. https://doi.org/10.1093/molbev/msh097

Liu, C., Worthington, R.J., Melander, C., Wu, H., 2011. A new small molecule specifically inhibits the cariogenic bacterium Streptococcus mutans in multispecies biofilms. Antimicrob. Agents Chemother. 55, 2679-2687. https://doi.org/10.1128/AAC.01496-10

Madeira, F., Park, Y.M., Lee, J., Buso, N., Gur, T., Madhusoodanan, N., Basutkar, P., Tivey, A.R.N., Potter, S.C., Finn, R.D., Lopez, R., 2019. The EMBL-EBI search and sequence analysis tools APIs in 2019. Nucleic Acids Res. 47, W636-W641. https://doi.org/10.1093/nar/gkz268

Mascher, T., Helmann, J.D., Unden, G., 2006. Stimulus Perception in Bacterial SignalTransducing Histidine Kinases. Microbiol. Mol. Biol. Rev. 70, 910-938. https://doi.org/10.1128/mmbr.00020-06

Ng, W.-L., Bassler, B.L., 2009. Bacterial Quorum-Sensing Network Architectures. Annu. Rev. Genet. 43, 197-222. https://doi.org/10.1146/annurev-genet-102108-134304

Papenfort, K., Bassler, B.L., 2016. Quorum sensing signal-response systems in Gram-negative bacteria. Nat. Rev. Microbiol. 14, 576-588. https://doi.org/10.1038/nrmicro.2016.89

Pearson, W.R., 2013. Selecting the Right Similarity-Scoring Matrix. Curr Protoc Bioinforma. 43, 3.5.1-3.5.9. https://doi.org/10.1002/0471250953.bi0305s43 
833

834

835

836

837

838

839

840

841

842

843

844

845

846

847

848

849

850

851

852

853

854

855

856

857

858

859

860

861

862

863

864

865

866

867

868

869

Rasmussen, B.B., Nielsen, K.F., Machado, H., Melchiorsen, J., Gram, L., Sonnenschein, E.C., 2014. Global and Phylogenetic Distribution of Quorum Sensing Signals, Acyl Homoserine Lactones, in the Family of Vibrionaceae. Mar. Drugs 12, 5527-5546. https://doi.org/10.3390/md12115527

Reading, N.C., Sperandio, V., 2006. Quorum sensing: The many languages of bacteria. FEMS Microbiol. Lett. 254, 1-11. https://doi.org/10.1111/j.1574-6968.2005.00001.x

Rutherford, S.T., Bassler, B.L., 2012. Bacterial quorum sensing: Its role in virulence and possibilities for its control. Cold Spring Harb. Perspect. Med. 2:a012427. https://doi.org/10.1101/cshperspect.a012427

Scoffone, V.C., Trespidi, G., Chiarelli, L.R., Barbieri, G., Buroni, S., 2019. Quorum sensing as antivirulence target in cystic fibrosis pathogens. Int. J. Mol. Sci. 20. https://doi.org/10.3390/ijms20081838

Sreenivasulu, Y., 2015. THE POTENTIAL OF MICROBIAL WEALTH FOR PHYTOPATHOGEN MANAGEMENT AND PLANT GROWTH ENHANCEMENT. J. Biol. Nat. 2(1), 6-15.

Stamatakis, A., 2014. RAxML version 8: A tool for phylogenetic analysis and post-analysis of large phylogenies. Bioinformatics 30, 1312-1313. https://doi.org/10.1093/bioinformatics/btu033

Sully, E.K., Malachowa, N., Elmore, B.O., Alexander, S.M., Femling, J.K., Gray, B.M., DeLeo, F.R., Otto, M., Cheung, A.L., Edwards, B.S., Sklar, L.A., Horswill, A.R., Hall, P.R., Gresham, H.D., 2014. Selective Chemical Inhibition of agr Quorum Sensing in Staphylococcus aureus Promotes Host Defense with Minimal Impact on Resistance. PLoS Pathog. 10. https://doi.org/10.1371/journal.ppat.1004174

Tiwari, R., Karthik, K., Rana, R., Malik, Y.S., Dhama, K., Joshi, S.K., 2016. Quorum sensing inhibitors/antagonists countering food spoilage bacteria-need molecular and pharmaceutical intervention for protecting current issues of food safety. Int. J. Pharmacol. 12, 262-271. https://doi.org/10.3923/ijp.2016.262.271

Wang, W., Shu, D., Chen, L., Jiang, W., Lu, Y., 2009. Cross-talk between an orphan response regulator and a noncognate histidine kinase in streptomyces coelicolor. FEMS Microbiol. Lett. 294, 150-156. https://doi.org/10.1111/j.1574-6968.2009.01563.x

West, A.H., Stock, A.M., 2001. Histidine kinases and response regulator proteins in twocomponent signaling systems. Trends Biochem. Sci. 26, 369-376. https://doi.org/10.1016/S0968-0004(01)01852-7

Whiteley, M., Diggle, S.P., Greenberg, E.P., 2017. Progress in and promise of bacterial quorum sensing research. Nature 551, 313-320. https://doi.org/10.1038/nature24624

Wilke, K.E., Francis, S., Carlson, E.E., 2015. Inactivation of multiple bacterial histidine kinases by targeting the ATP-binding domain. ACS Chem. Biol. 10, 328-335. https://doi.org/10.1021/cb5008019 
bioRxiv preprint doi: https://doi.org/10.1101/2020.11.15.381053; this version posted March 16, 2021. The copyright holder for this preprint (which was not certified by peer review) is the author/funder, who has granted bioRxiv a license to display the preprint in perpetuity. It is made available under aCC-BY-NC 4.0 International license.

870 Worthington, R.J., Blackledge, M.S., Melander, C., 2013. Small-molecule inhibition of 871 bacterial two-component systems to combat antibiotic resistance and virulence. Future 872 Med. Chem. 5, 1265-1284. https://doi.org/10.4155/fmc.13.58

873

874 
bioRxiv preprint doi: https://doi org/10.1101/202011.15.381053: this version posted March 16,2021 . The copyright holder for this preprint (which was not certified by peer review) is the author/funder, who has granted bioRxiv a license to display the preprint in perpetuity. It is made available under aCC-BY-NC 4.0 International license.

\section{$875 \quad$ Figure and table legends}

876

877 Figure 1: Presence of the protein subunits of different QS pathways in selected

878 groups of bacteria. Colored sectors indicate presence, empty sectors indicate

879 absence, based on the KEGG taxonomy database for each orthology group. "Other"

880 Spirochaetes refer toTreponema, Spirochaeta, Leptospira and Salinispira.

881

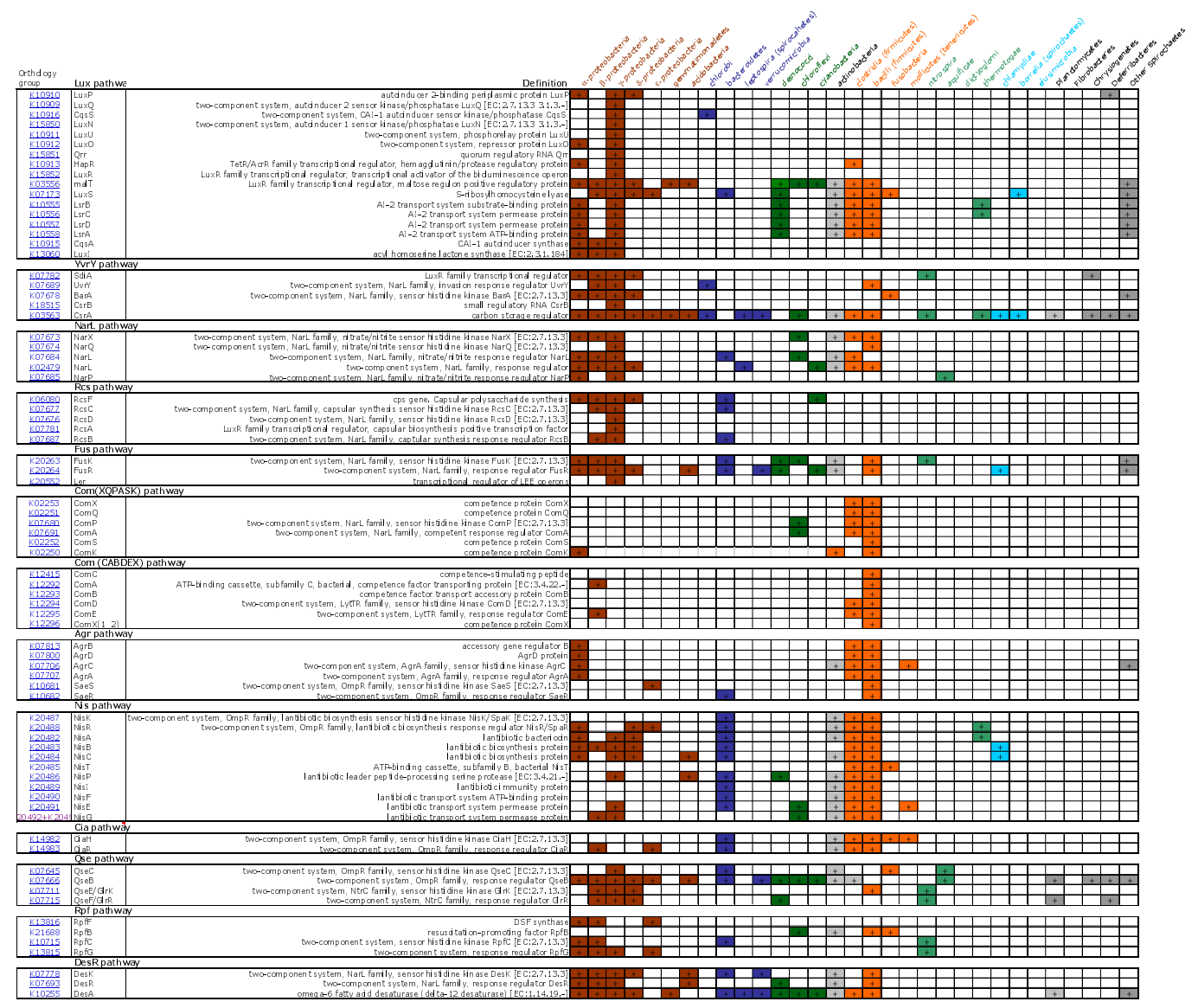

883

884 
885 Figure 2: The number of bacterial groups in which each protein is found. The

886 different colors indicate the family of each HK and RR in our study; proteins involved

887 in the same pathway are indicated with a lighter shade of the same color. Blue: Lux 888 family, Green: NarL family, Yellow: LytTR family, Orange: OmpR family, Purple: NtrC 889 family, Grey: "Other", Black: Des pathway.

890

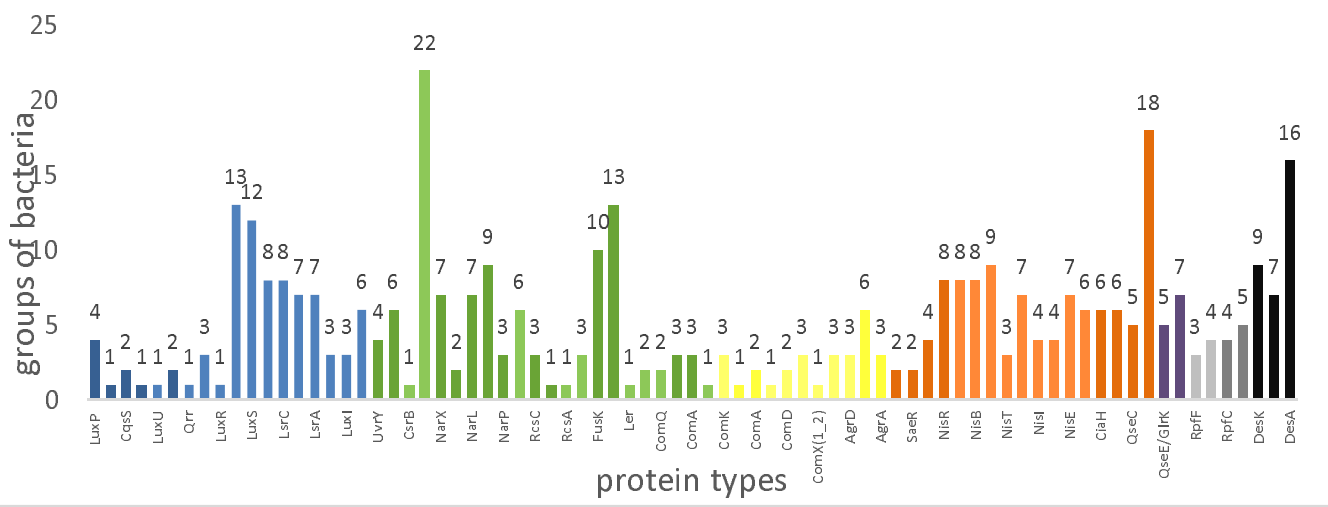

891

892 
Figure 3: An overview of the position of the conserved domains in the HKs and RRs of this study. The similar colors of domains indicate their presence in a common clan of the Pfam database. The pathway drawings are from KEGG PATHWAY map ko02020, edited with Microsoft Draw.
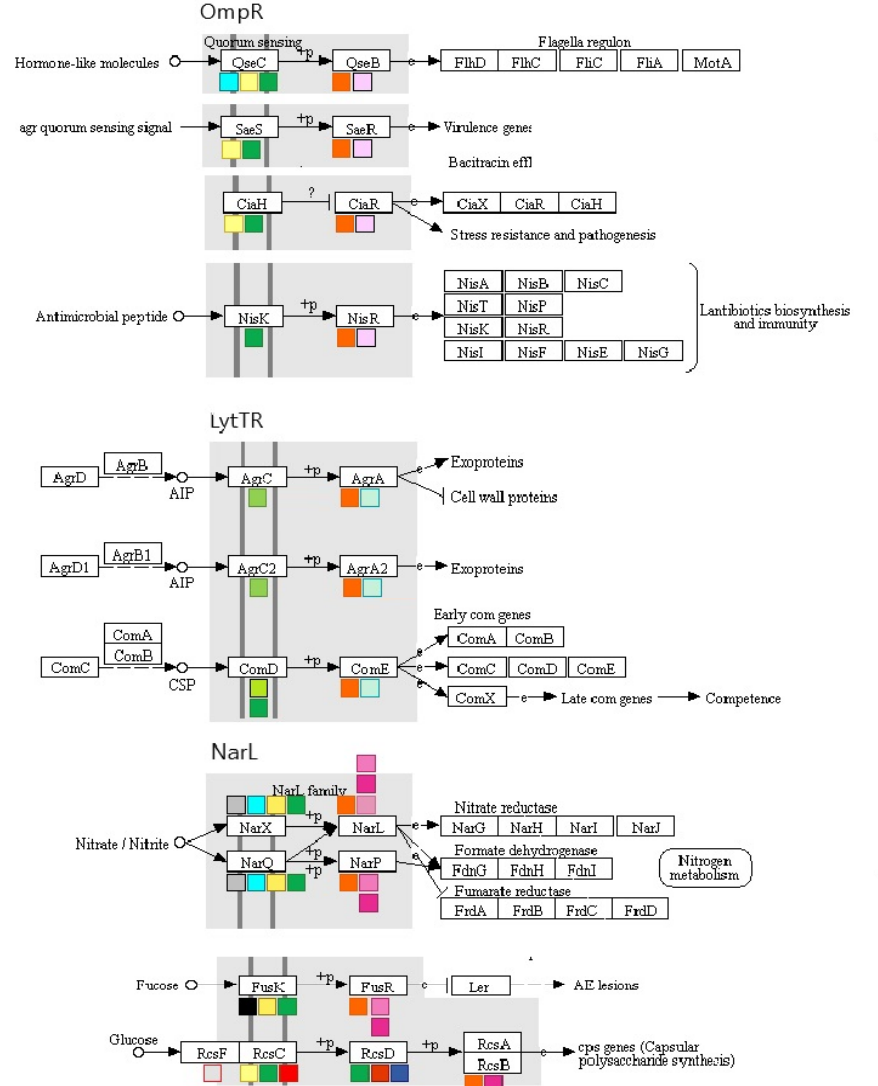

$\longrightarrow \longrightarrow$ SdiA
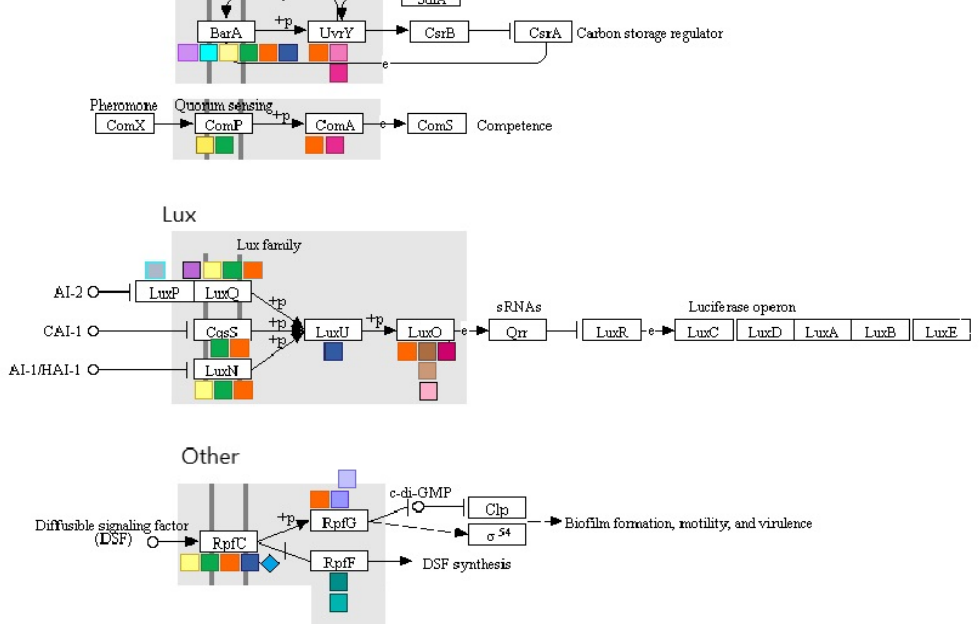

NtrC

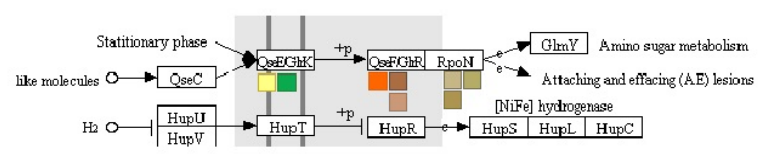

$\begin{array}{ll}\square \text { 2CSK_N } & \square \text { HTH_8 } \\ \square \text { PilJ } & \text { Sigma54_activ_2 } \\ \square \text { DUF2222 } & \text { GerE } \\ \square \text { HisKA } & \text { Sigma70_r4 } \\ \square \text { HisKA_3 } & \text { Sigma70_r4_2 } \\ \square \text { Response_reg } & \square \text { Sigma54_activat } \\ \square \text { Hpt } & \square \text { Sigma54_CBD } \\ \square \text { HAMP } & \square \text { Sigma54_DBD } \\ \square \text { HATPase_c } & \square \text { Sigma54_AID } \\ \square \text { HATPase_c_5 } & \square \text { Trans_reg_C } \\ \square \text { AAA } & \square \text { LytTR } \\ \square \text { AAA_5 } & \square \text { HD } \\ \square \text { MASE_1 } & \square \text { HD_5 } \\ \square \text { RcsD_ABL } & \square \text { ECH_1 } \\ \square \text { RcsC } & \square \text { ECH_2 } \\ \square \text { LuxQ_periplasm } & \square \text { RcsF } \\ \square \text { Peripla_BP } & \end{array}$

$\square$ 2CSK_N $\square$ HTH_8

- GerE

Sigma70_r4

- Sigma70_r4_2

- Sigma54_activat

- Sigma54_CBD

$\square$ Trans_reg_C

$\square$ LytTR

$\square$ ECH_1

$\square \mathrm{ECH} 2$

- Peripla_BP 
898 Figure 4: Phylogenetic analysis of the NarL family. (A) Histidine Kinases. (B) 899 Response Regulators. Both trees reconstructed using PhyML based on the MAFFT

900 alignment. The color of the bullets indicates the bootstrap value of the nodes. White:

$90150 \%-80 \%$, Grey: 80\%-95\%, Black: over 95\%

902

903 Figure 5: Phylogenetic analysis of the OmpR family. (A) Histidine Kinases. (B) 904 Response Regulators. Both trees reconstructed using PhyML based on the MAFFT 905 alignment. The color of the bullets indicates the bootstrap value of the nodes, as in 906 Figure 4.

907

908 Figure 6: Phylogenetic analysis of the Lux family histidine kinases. Tree 909 reconstructed using PhyML based on the MAFFT alignment. The color of the bullets 910 indicates the bootstrap value of the nodes, as in Figure 4.

911

912 Figure 7: Phylogenetic analysis of the LytR family. (A) Histidine Kinases. (B) 913 Response Regulators. Both trees reconstructed using PhyML based on the MAFFT 914 alignment. The color of the bullets indicates the bootstrap value of the nodes, as in 915 Figure 4.

917 Figure 8: Phylogenetic analysis of all histidine kinase families. Tree 918 reconstructed using RaxML based on the MAFFT alignment. The bullets indicate the 919 bootstrap value of the nodes, as in Figure 4.

920

921 Figure 9: Phylogenetic analysis of all response regulator families. Tree 922 reconstructed using RaxML based on the MAFFT alignment. The bullets indicate the 923 bootstrap value of the nodes, as in Figure 4.

924 
926 Table 1: Categorization of the conserved domains of all the HK and RR types in

927 this study.

928

\begin{tabular}{|c|c|c|c|c|c|c|}
\hline Clans & & & Domains & & & \\
\hline $\begin{array}{l}\text { HTH (Helix- } \\
\text { Turn-Helix) }\end{array}$ & HTH_8 & Sigma70_r4 & $\begin{array}{l}\text { Sigma70_r4 } \\
2\end{array}$ & GerE & $\begin{array}{l}\text { Sigma54 } \\
\text { CBD }\end{array}$ & $\begin{array}{l}\text { Sigma54 } \\
\text { DBD }\end{array}$ \\
\hline His kinase $\mathrm{A}$ & HATPase_c & HATPase_c_5 & HisKA & HisKA_3 & & \\
\hline $\begin{array}{l}\text { P-loop- } \\
\text { NTPase }\end{array}$ & AAA & AAA_5 & $\begin{array}{l}\text { Sigma54_ac } \\
\text { tivitat }\end{array}$ & $\begin{array}{l}\text { Sigma54 } \\
\text { activ_2 }\end{array}$ & & \\
\hline $\begin{array}{l}\text { Cache-like } \\
\text { domains }\end{array}$ & 2CSK_N & DUF2222 & $\begin{array}{l}\text { LuxQ- } \\
\text { periplasm } \\
\text { periplasmic } \\
\text { sensor } \\
\text { domain }\end{array}$ & & & \\
\hline $\begin{array}{l}\text { Chey-like } \\
\text { superfamily }\end{array}$ & $\begin{array}{l}\text { Response_reg } \\
\text { Receiver } \\
\text { domain of RRs }\end{array}$ & $\begin{array}{l}\text { RcsD_ABL } \\
\text { Found in the } \\
\text { RcsD HK, } \\
\text { recognizes }\end{array}$ & $\mathrm{RcsC}$ & & & \\
\hline & & $\begin{array}{l}\text { the RcsB } \\
\text { effector } \\
\text { domain. }\end{array}$ & & & & \\
\hline HD-PDEase & HD & HD_5 & & & & \\
\hline $\begin{array}{l}\text { Clp } \\
\text { crotonase }\end{array}$ & $\begin{array}{l}\text { ECH_1 } \\
\text { Enoyl-CoA } \\
\text { hydratase }\end{array}$ & $\begin{array}{l}\text { ECH_2 } \\
\text { (Enoyl-CoA } \\
\text { hydratase }\end{array}$ & & & & \\
\hline Periplas BP & Peripla_BP & & & & & \\
\hline HAMP & HAMP & & & & & \\
\hline Tudor & $\begin{array}{l}\text { LytTR } \\
\text { DNA-binding } \\
\text { domain }\end{array}$ & & & & & \\
\hline YbjQ-like & $\begin{array}{l}\text { RcsF } \\
\text { lipoprotein }\end{array}$ & & & & & \\
\hline 929 & & & & & & \\
\hline 930 & & & & & & \\
\hline 931 & & & & & & \\
\hline 932 & & & & & & \\
\hline
\end{tabular}


933 Table 2: Occurence of the conserved domains of all the HKs and RRs in the 934 different protein types and families.

935

\begin{tabular}{lll}
\hline Domain & Protein Types & Family \\
\hline HATPase_c & 15 & All of the families \\
HATPase_c_5 & 3 & LytTR \\
HisKA & 9 & OmpR, NarL, Lux, Other, NtrC \\
HisKA_3 & 4 & NarL \\
HAMP & 3 & NarL \\
Response_reg & 3 & NarL, Lux, Other \\
Hpt & 2 & NarL, Other \\
Trans_reg_C & 4 & OmpR \\
LytTR & 3 & LytTR \\
GerE & 5 & NarL \\
Sigma70_r4_2 & 4 & NarL \\
Sigma54_activat & 2 & LuxO, NtrC \\
Sigma54_activ_2 & 2 & LuxO, NtrC
\end{tabular}


A

ComP

ResC

BarA
FusK

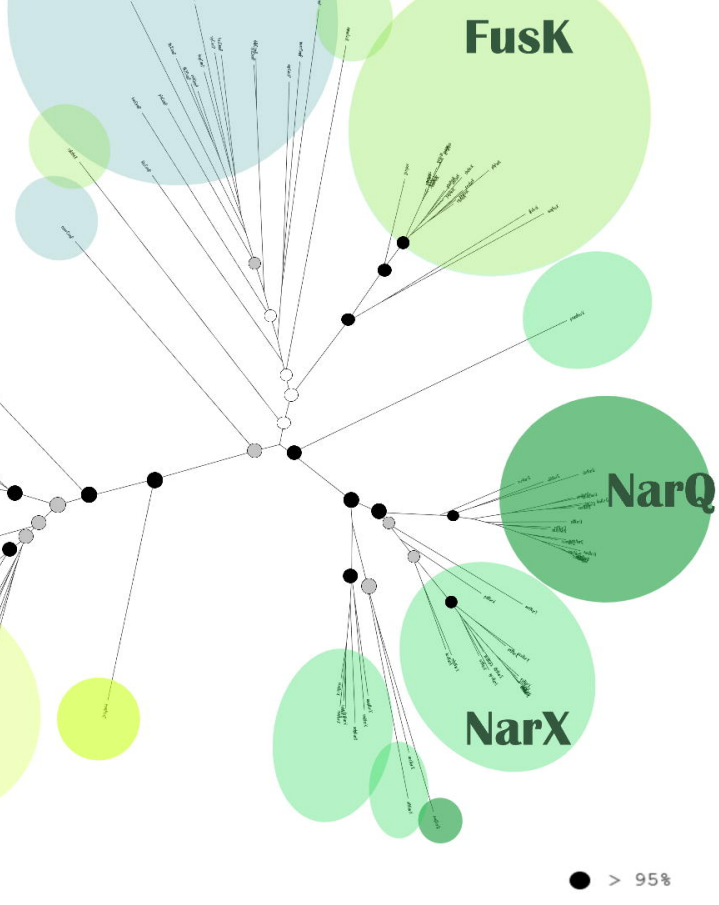

- $80 \%-95 \%$

- 50 응 -80 웅
B

RcsB

NarL

\section{FusR}

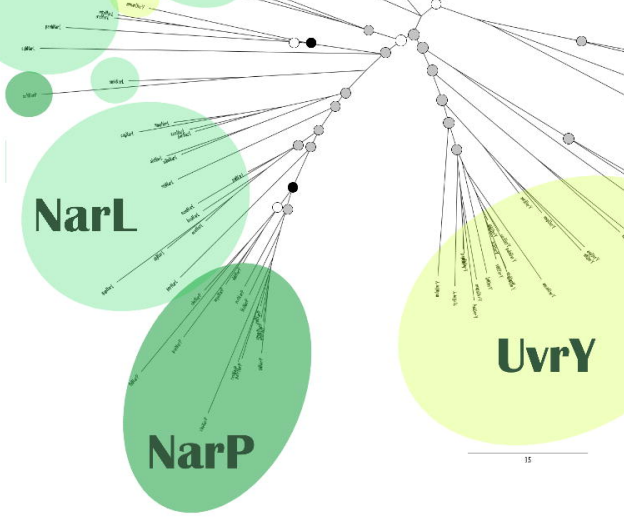

UvrY

80\%-95\%

O 50 z $-80 \%$ 
A

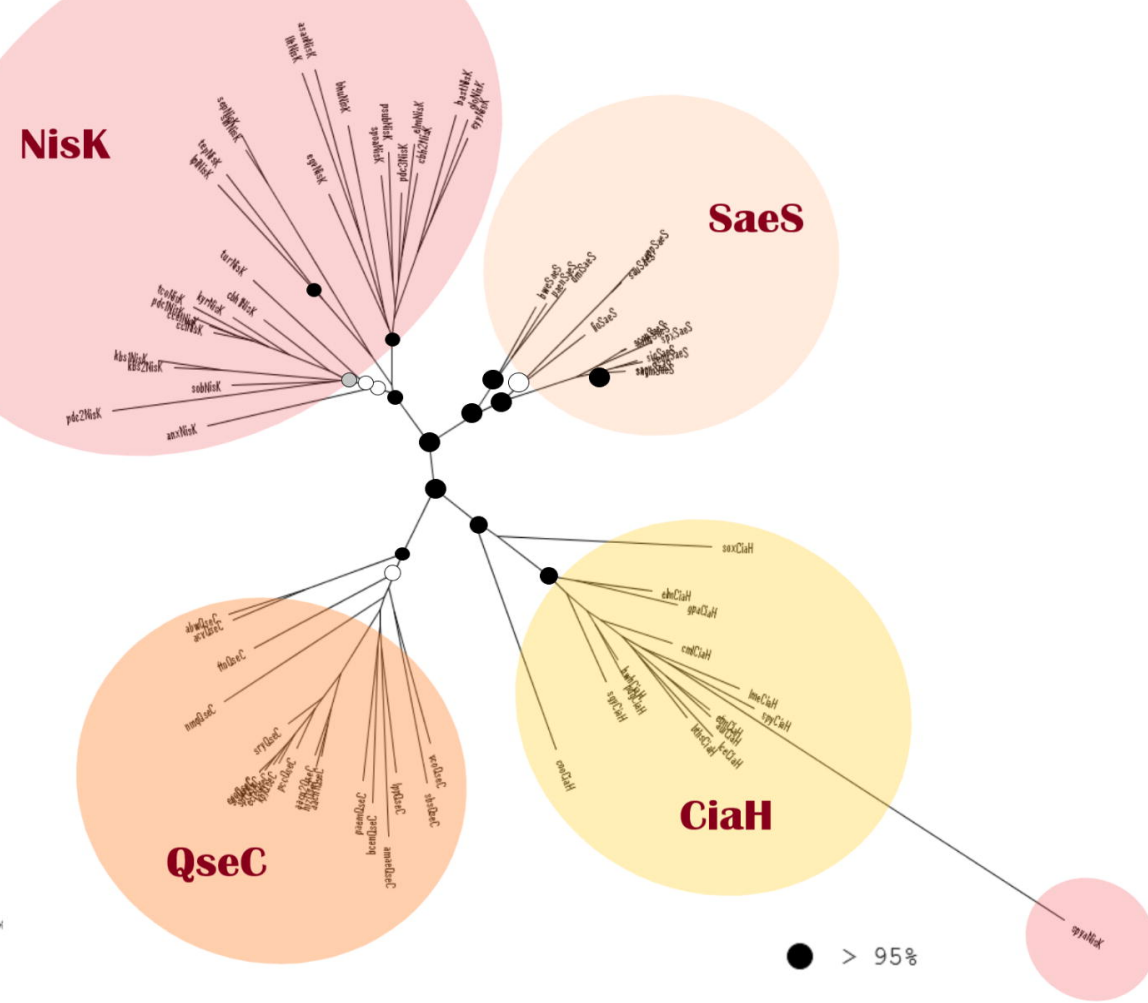

B

NisR

Y , SaeR

CiaR

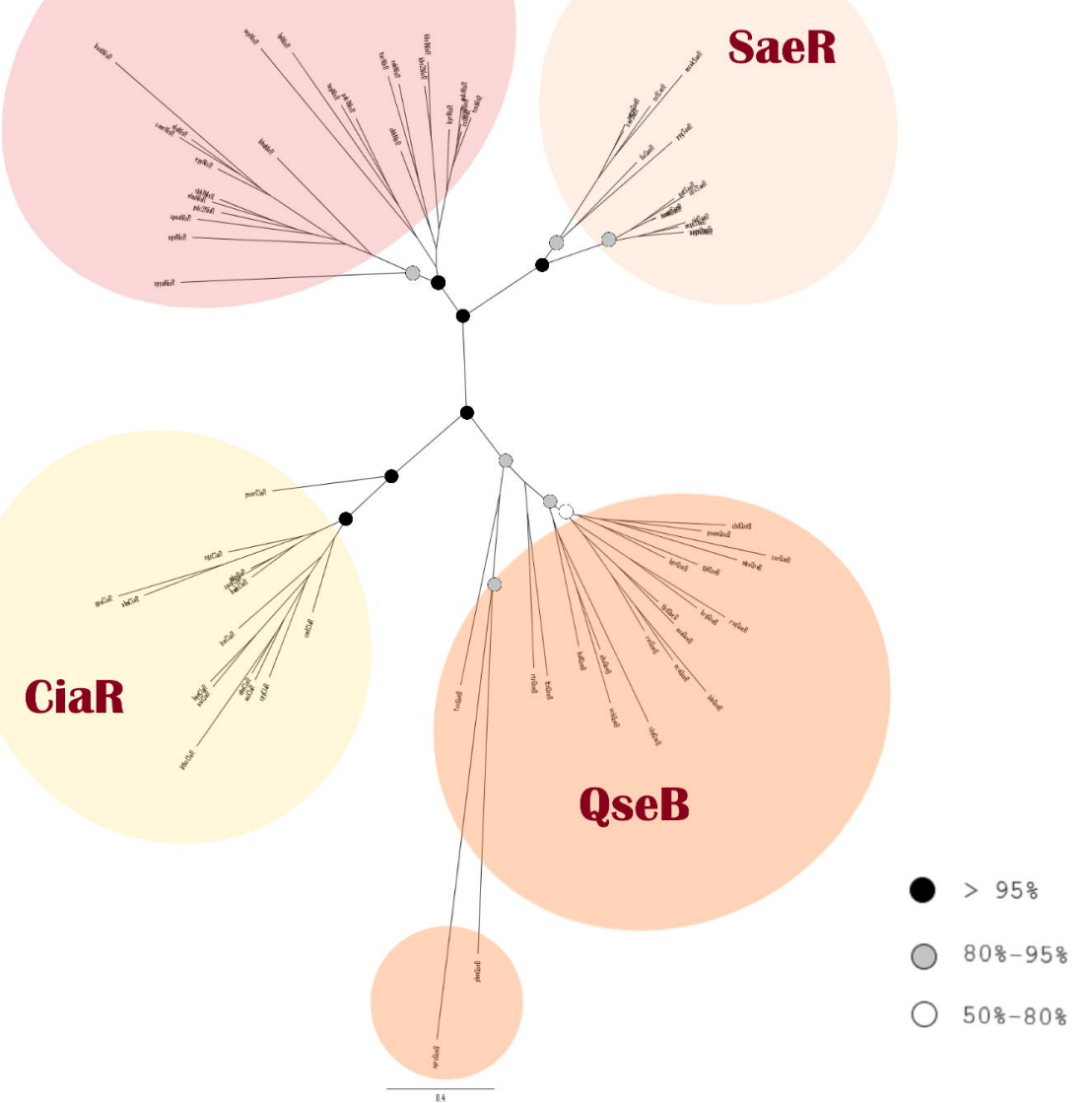




\section{LuxQ}

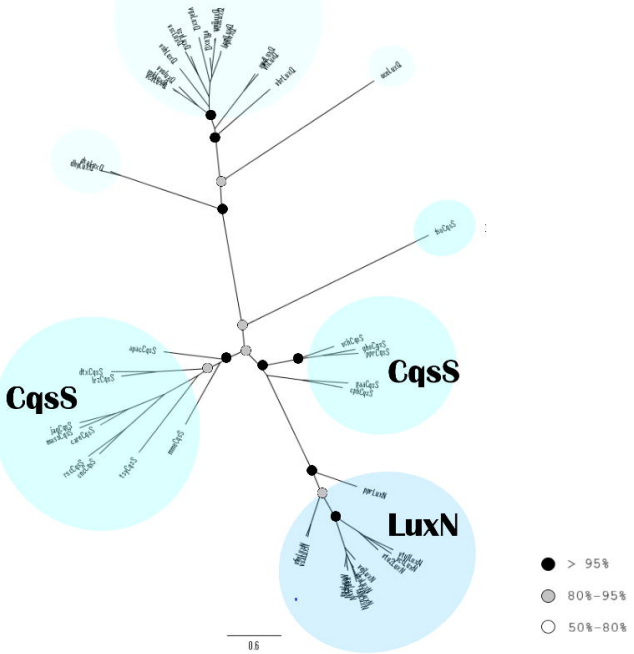




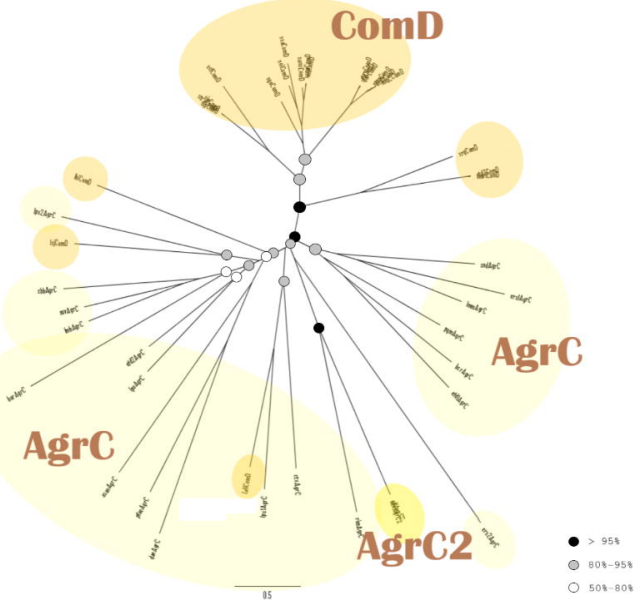

\section{ComE}

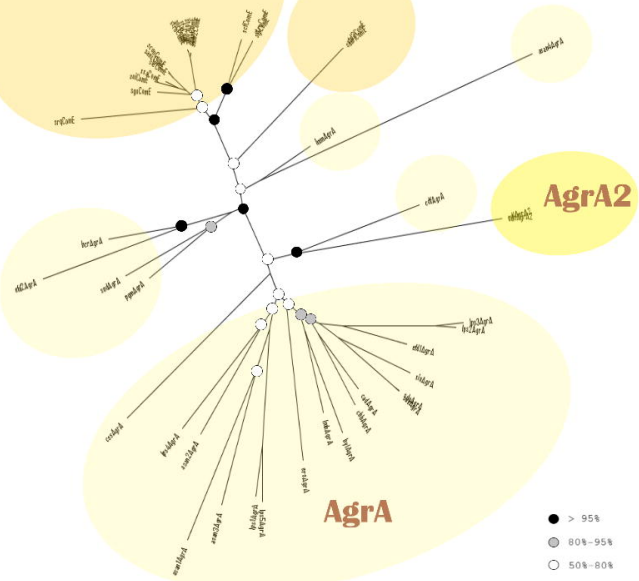




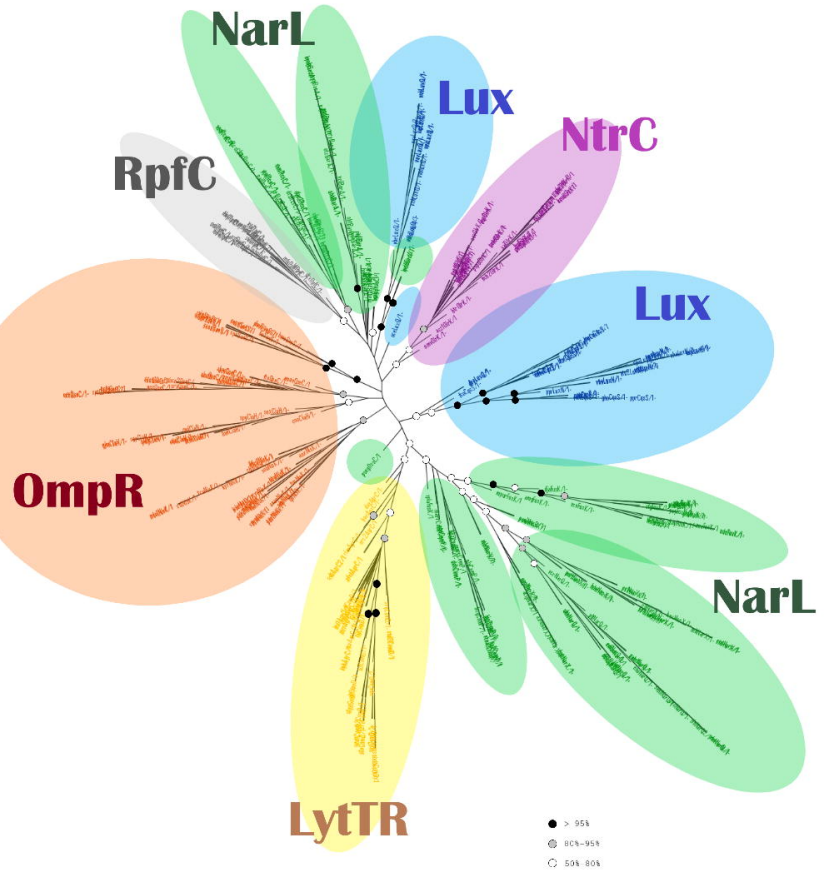




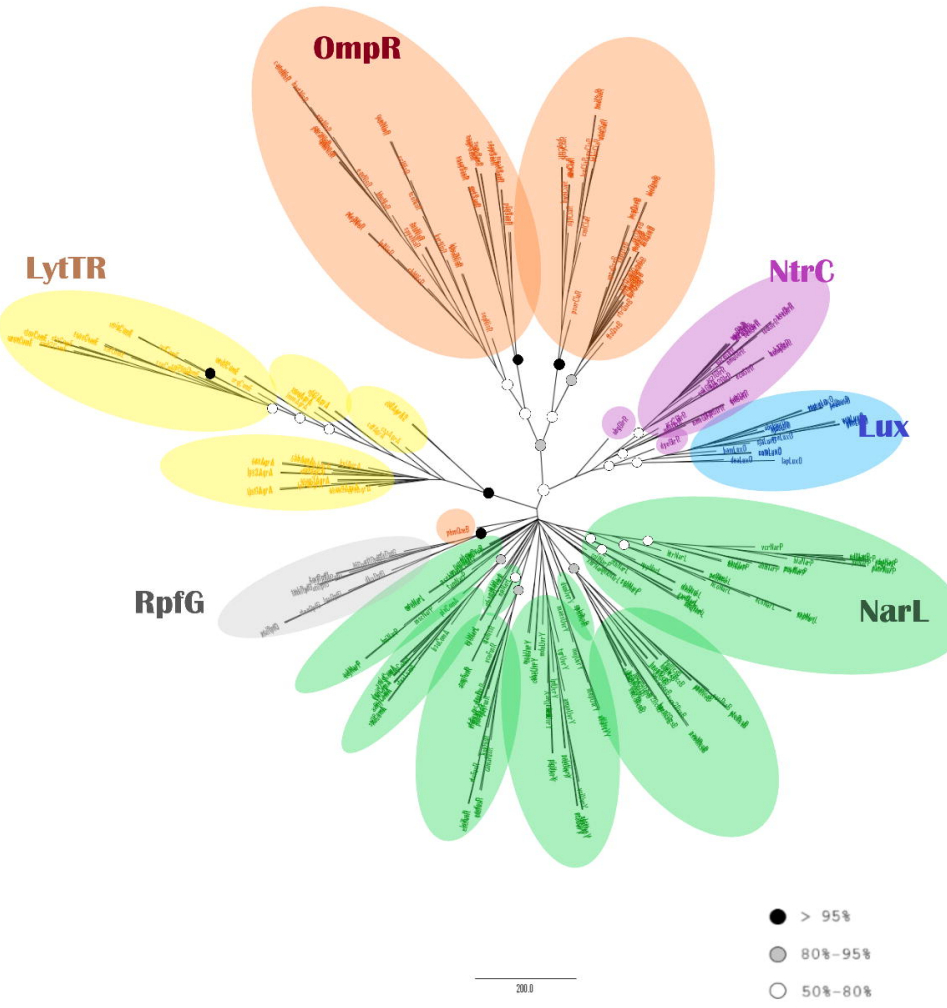

